Original Research Paper

\title{
Micro-macro homogenization of granular materials based on the average-field theory of Cosserat continuum
}

\author{
Qipeng Liu ${ }^{\mathrm{a}, *}$, Xiaoyu Liu ${ }^{\mathrm{b}}$, Xikui $\mathrm{Li}^{\mathrm{c}}$, Shihai $\mathrm{Li}^{\mathrm{b}}$ \\ ${ }^{a}$ School of Civil and Safety Engineering, Dalian Jiaotong University, Dalian 116028, PR China \\ ${ }^{\mathrm{b}}$ Institute of Mechanics, Chinese Academy of Sciences, Beijing 100190, PR China \\ ${ }^{\mathrm{c}}$ The State Key Laboratory for Structural Analysis of Industrial Equipment, Dalian University of Technology, Dalian 116024, PR China
}

\section{A R T I C L E I N F O}

Article history:

Received 2 February 2013

Received in revised form 4 July 2013

Accepted 8 July 2013

Available online 24 July 2013

\section{Keywords:}

Granular materials

Average-field theory

Effective couple stress tensor

Micromechanically based constitutive

models

\begin{abstract}
A B S T R A C T
This paper performs further study on the micro-macro homogenization approach of granular materials (Li et al., 2010) based on the advancement of Hill's lemma for Cosserat continuum (Liu, 2013). Firstly, the average couple stress tensor, expressed as the volume integration of quantities over the representative volume element (RVE) in the average-field theory of Cosserat continuum, is further deduced and expressed in terms of discrete quantities on the discrete particle assembly RVE of granular materials. The expression is also discussed and compared with other typical definitions of the effective couple stress tensor for granular materials in the literature. Then, rate forms of micromechanically based constitutive models consistent with different types of RVE boundary conditions are derived and discussed. Since the presented micro-macro homogenization approach is used, not only the micro-macro energy equivalence is satisfied, but also the microstructure and its evolution can be taken into account in the constitutive formulation with no need of specifying macroscopic phenomenological constitutive model.
\end{abstract}

(C) 2013 The Society of Powder Technology Japan. Published by Elsevier B.V. and The Society of Powder Technology Japan. All rights reserved.

\section{Introduction}

Most engineering materials, such as composites and granular materials are heterogeneous in nature. It is of great importance to determine the micromechanically based macroscopic overall characteristics of these materials for engineering applications. The average-field theory is one of the basic approaches in micromechanics dealing with this subject. This theory is based on the fact that the effective mechanical properties are determined by relations between the volume averages of the stresses and strains of microscopically heterogeneous samples. Hence, macroscopic-field quantities are defined as the volume averages of the corresponding microscopic-field ones, and the effective properties are determined by relations between the averaged microscopic-field quantities under the prescribed boundary conditions on the representative volume element (RVE). The average-field theory of the classical continuum has been well developed and widely applied to the multi-scale analysis and computation of composites. An abundant literature, including the work contributed by Hill [1], Hashin [2], Suquet [3], Nemat-Nasser and Hori [4], Michel et al. [5], Miehe and Koch [6], Qu and Cherkaoui [7], deals with this subject.

\footnotetext{
* Corresponding author. Tel./fax: +86 41184105356.

E-mail address: q.liu@hotmail.com (Q. Liu).
}

Granular material is a typical heterogeneous medium and consists of discrete grains and voids. This discontinuous medium is represented by an equivalent continuum on the macroscopic level. The phenomenological constitutive models are needed to describe the mechanical behavior. These models are usually complex and contain some fitting parameters which are difficult to be determined. On the other hand, granular material is naturally modeled as an assembly of discrete particles in contact from the microscopic view. However, if a typical macroscopic structure is fully described as a discrete particle assembly, the computation task is too heavy to be acceptable. Therefore, it is reasonable and significant to develop proper multi-scale homogenization schemes combining the macroscopic and microscopic models.

One major objective of the multi-scale analysis for granular materials is to determine the effective overall properties and responses of the heterogeneous medium in terms of the properties and responses of the microstructure $[8,9]$. The nature of granular medium is that each discrete particle possesses rotational degrees of freedom in addition to translational degrees of freedom in the kinematics and is capable of bearing and transmitting couples in addition to forces from one particle to the other in contact in the kinetics $[10,11]$. Thus, the RVE, considered as a discrete particle assembly characterizing the local microstructure, should be modeled as an equivalent Cosserat continuum [12-15] instead of a classical continuum in order to link with the macroscopic Cosserat continuum. This process implies that Cosserat continuum is 
assumed at both microscopic and macroscopic scales in the homogenization procedures. Cosserat continuum model has been successfully applied to the simulation of phenomena with size effect, which is essentially a multi-scale phenomenon and stems from the influence of the microscopic structures on the macroscopic behavior [16-20]. However, fewer contributions have been devoted to the development of the homogenization scheme for the heterogeneous Cosserat continuum in contrast to the case in classical continuum. Among them are Forest et al. [21], Yuan and Tomita [22], Hu et al. [23], Sulem and Muhlhaus [24], Trovalusci and Masiani [25], and Besdo [26].

A micro-macro homogenization approach for discrete particle assembly - Cosserat continuum modeling of granular materials is proposed in the framework of the average-field theory [27]. The interest lies in that the constitutive relation is formulated to take into account the microstructure and its evolution with no need of specifying macroscopic phenomenological constitutive model. Starting from the derivation of a version of Hill's lemma for Cosserat continuum, proper RVE boundary conditions connecting the micro and macro quantities are determined. With the link between the discrete particle assembly and its equivalent Cosserat continuum in an individual RVE, the boundary conditions prescribed on the RVE modeled as Cosserat continuum can be transformed into those prescribed on the peripheral particles of the RVE modeled as the discrete particle assembly. The average stress and average couple stress measures are further expressed in terms of the integrations along the boundary of the RVE modeled as a Cosserat continuum. These measures are then determined by using the discretized counterpart of the boundary integrations evaluated at the discrete contacting points of the peripheral particles in the assembly. Finally, the rate forms of micromechanically based macroscopic constitutive relation taking into account the microstructure and its evolution is formulated in light of proper RVE boundary conditions and the definitions of corresponding macroscopic average quantities.

Although the primary idea and procedure of the proposed approach are formulated in paper [27], some basic issues are still open and need to be further elaborated. On the one hand, the expression of the macroscopic average couple stress tensor in terms of microscopic stresses and/or couple stresses is not unique in the average-field theory of Cosserat continuum [10,14,21-23]. There are mainly two kinds of expression used in the literature, i.e. $\bar{\mu}_{i j}=\frac{1}{V} \int_{V} \mu_{i j} d V$ suggested by Yuan and Tomita [22] and $\bar{\mu}_{i j}=\frac{1}{V} \int_{V}\left(\mu_{i j}-x_{i} e_{j k l} \sigma_{k l}\right) d V$ proposed by Forest et al. [21]. Only the former definition $\bar{\mu}_{i j}=\frac{1}{V} \int_{V} \mu_{i j} d V$ was adopted to derive Hill's lemma of Cosserat continuum and to develop the micro-macro homogenization scheme of granular materials in paper [27]. On the other hand, only mixed translational displacement-surface couple conditions can be prescribed on the RVE boundary according to the Hill's lemma in paper [27]. Other types of RVE boundary conditions commonly used in classical homogenization method were not properly given for Cosserat continuum. It should be stressed that the above two issues are vital to the homogenization approach of granular materials in order to ensure the satisfaction of the micro-macro energy equivalence (named 'Hill-Mandel condition') and to derive the micromechanically based constitutive models. Recent research on the average-field theory of Cosserat continuum produces a new version of Hill's lemma based on the definition $\bar{\mu}_{i j}=\frac{1}{V} \int_{V}\left(\mu_{i j}-x_{i} e_{j k l} \sigma_{k l}\right) d V$, from which more versatile RVE boundary conditions are properly given [28]. This motivates us to reconsider and extend the homogenization approach of granular materials proposed in paper [27].

This paper performs further study on the micro-macro homogenization scheme of granular materials based on the development of Hill's lemma for Cosserat continuum. Firstly, the average couple stress tensor, expressed as the volume integration of quantities over the RVE in the average-field theory of Cosserat continuum [28], is deduced and expressed in terms of quantities on the RVE boundary. The definition can be further expressed in terms of static quantities over the domain of the discrete particle assembly RVE of granular materials. The significance for the definition of the effective couple stress tensor of granular materials is presented by comparison with other typical definitions in the literature. Then, rate forms of micromechanically based constitutive models for granular materials are derived according to three types of RVE boundary conditions in the strong form, i.e. homogeneous translational and rotational displacement boundary conditions, mixed translational displacement and surface couple boundary conditions, and mixed surface traction and rotational displacement boundary conditions. Further analysis and discussion are also given on these constitutive models. Compared to the previous contribution [27], this paper presents a more comprehensive theoretical reference for the micro-macro homogenization of discrete particle assembly - Cosserat continuum modeling in the framework of the average-field theory.

\section{Cosserat theory: equilibrium and kinematics}

In a Cosserat model the independent kinematical degrees of freedom are the displacements $u_{i}$ and the rotations $\omega_{i}$. Accordingly, in addition to the classical stresses $\sigma_{i j}$, independent couple stresses $\mu_{i j}$ are introduced [16-20], as shown in Fig. 1. The strain measures $\varepsilon_{i j}$ and $\kappa_{i j}$ (curvatures) are defined as

$\varepsilon_{i j}=u_{j, i}-e_{i j k} \omega_{k}$

$\kappa_{i j}=\omega_{j, i}$

where $e_{i j k}$ is the permutation tensor. The summation rule of tensor indices will be applied throughout the present paper. The equilibrium conditions can be expressed as

$\sigma_{i j, i}=0$

$\mu_{i j, i}+e_{j k l} \sigma_{k l}=0$

Equilibrium on the surface of the Cosserat RVE requires that

$t_{i}=n_{j} \sigma_{j i}$

$q_{i}=n_{j} \mu_{j i}$

where $t_{i}$ is the surface traction, $q_{i}$ is the surface couple, and $n_{j}$ is the unit vector normal to the surface.

\section{Hill's lemma and RVE boundary conditions of Cosserat continuum}

In this section, Hill's lemma and RVE boundary conditions in the average-field theory of Cosseat continuum in paper [28] are briefly

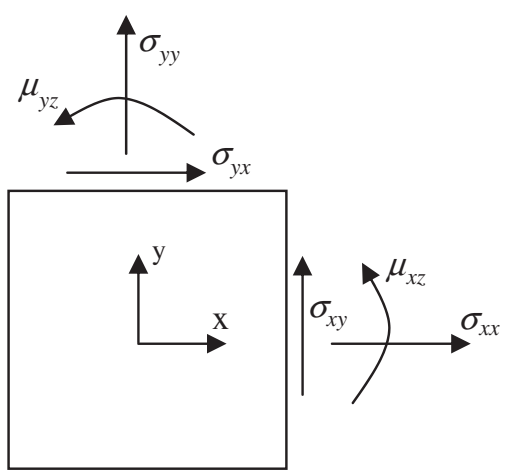

Fig. 1. Stresses and couple stresses for Cosserat continuum in 2-D case. 
listed. Besides, the signification of the average rotational degrees of freedom is further highlighted from the view of their higher order essence and the application to the fact of granular materials.

The micro-macro homogenization approach in the frame of average-field theory for heterogeneous materials can be schematically demonstrated by Fig. 2 . The concept of an RVE is used to estimate the continuum properties under specified boundary conditions determined by the deformation of macroscopic continuum point and its infinitesimal neighborhood. A typical RVE with volume $V$ and boundary $S$ is considered to be associated with a given sample point, which is usually an integration point of the macro-mesh. For any microscopic stress fields $\sigma_{i j}, \mu_{i j}$ and microscopic strain fields $\varepsilon_{i j}$, $\kappa_{i j}$ in the RVE under prescribed boundary traction $\left(t_{i}, q_{i}\right)$ or boundary displacement $\left(u_{i}, \omega_{i}\right)$ conditions, we denote the average stresses and the average strains over the domain of the RVE with $\bar{\sigma}_{i j}, \bar{\mu}_{i j}$ and $\bar{\varepsilon}_{i j}, \bar{\kappa}_{i j}$. Hereafter in this paper, as the over-bar is applied to a microscopic quantity $(*),(\bar{*})$ is defined to denote its volume average over the domain of the RVE. With this stipulation in symbol, according to the basic assumptions of the classical homogenization theory, we denote the macroscopic average strains and curvatures over the domain of the RVE as

$\bar{\varepsilon}_{i j}=\frac{1}{V} \int_{V} \varepsilon_{i j} d V=\frac{1}{V} \int_{V}\left(u_{j, i}-e_{i j k} \omega_{k}\right) d V=\frac{1}{V} \int_{V} u_{j, i} d V-e_{i j k} \frac{1}{V} \int_{V} \omega_{k} d V=\bar{u}_{j, i}-e_{i j k} \bar{\omega}_{k}$

$\bar{\kappa}_{i j}=\bar{\omega}_{j, i}=\frac{1}{V} \int_{V} \omega_{j, i} d V=\frac{1}{V} \int_{S} n_{i} \omega_{j} d S$

It should be pointed out that the volume average over the RVE is usually performed to the differential of the basic kinematical quantities in the average-field theory, for example $\bar{u}_{j, i}=\frac{1}{V} \int_{V} u_{j, i} d V$. Then, the volume average can be further expressed in terms of the integrations along the RVE boundary by use of Gauss theorem, for example $\bar{u}_{j, i}=\frac{1}{V} \int_{V} u_{j, i} d V=\frac{1}{V} \int_{S} n_{i} u_{j} d S$. However, Eq. (7) gives $\bar{\omega}_{k}=\frac{1}{V} \int_{V} \omega_{k} d V$ according to the definition of macroscopic strains $\bar{\varepsilon}_{i j}$, i.e., the volume average is directly performed to the rotational degrees of freedom. The reason lies in that the rotational degrees of freedom essentially belong to higher order terms compared to the translational degrees of freedom in continuum mechanics. This has been shown in the governing Eq. (1), in which the rotational degrees of freedom directly contribute to the strains. Thus we can conclude that the rotations belong to the order of the gradient of translational displacements. It has been pointed out that the continua with local rigid structure belong to the class of higher-order materials [29], and the strain gradient theory [30] gave the expression $\bar{\omega}_{i}=\frac{1}{2} e_{i j k} \bar{u}_{k, j}$, which also proved this point. Although the rotational degrees of freedom are independent of the translational degrees of freedom in Cosserat continuum model, the higher order essence of rotations compared to the translational displacements cannot be neglected in the formulation.

Besides, although the rotational degrees of freedom and corresponding intrinsic scale parameters have been successfully applied as a regularization mechanism in the macroscopic finite element simulation of the strain localization behavior of granular materials [16-20], the physical meaning of rotational degrees of freedom in Cosserat continuum model are still obscure for granular materials. The expression $\bar{\omega}_{k}=\frac{1}{V} \int_{V} \omega_{k} d V$ given in this paper can be further deduced as $\bar{\omega}=\frac{1}{V} \int_{V} \omega d V=\frac{1}{V} \sum_{i=1}^{N_{g}} \omega^{i} V^{i}$, in which $N_{g}$ is the total
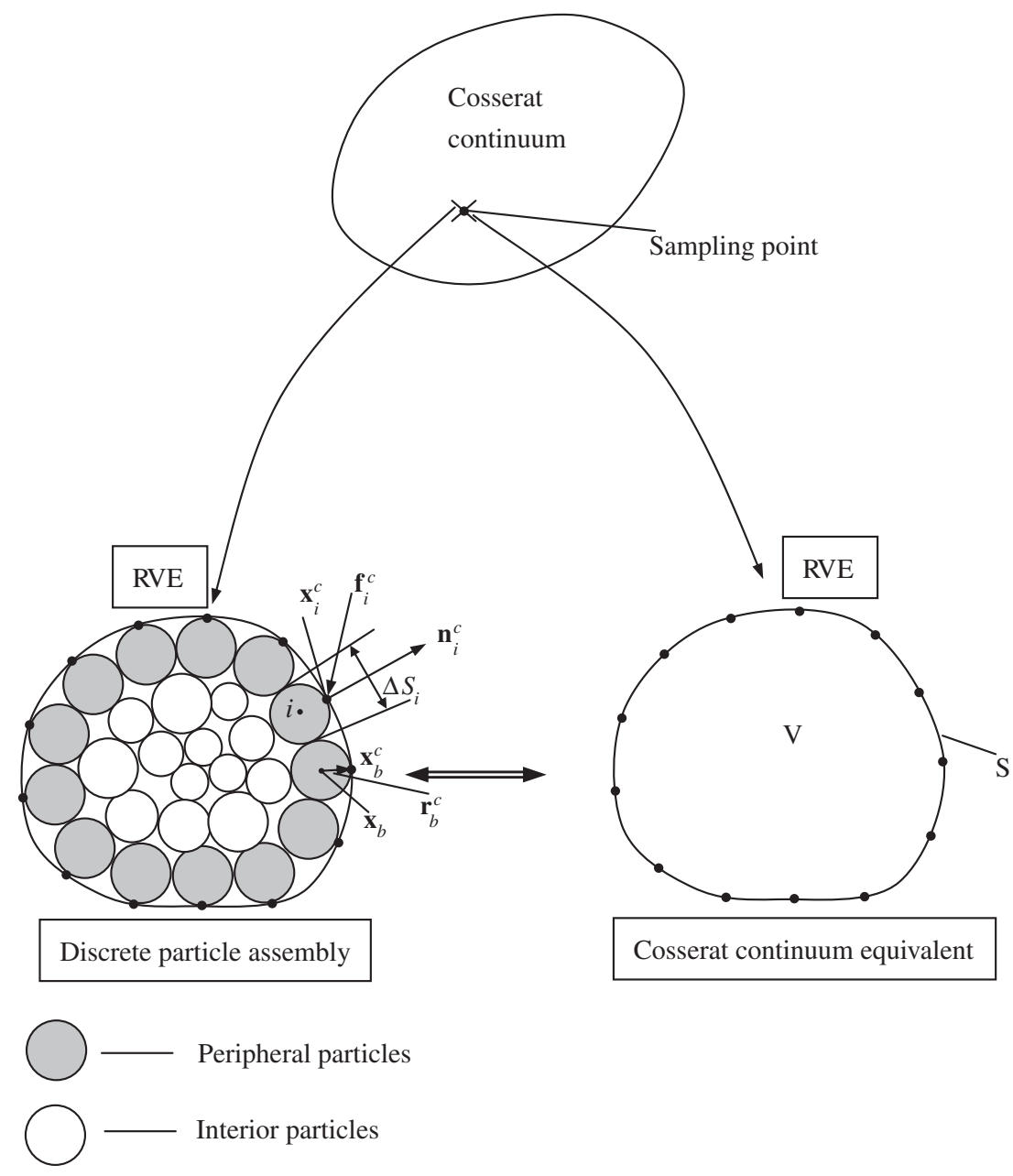

Fig. 2. Micro-macro homogenization of granular materials: discrete particle assembly - Cosserat continuum modeling. 
number of particles in the RVE, $\omega^{i}$ and $V^{i}$ is respectively the rotation and the volume of the $i$ 'th discrete particle in the RVE. It implies that, for granular materials, the macroscopic rotational degrees of freedom $\bar{\omega}_{k}$ are equal to the volume average of the independent rotational displacements of discrete particles over the microscopic RVE, which provides a physical interpretation for the rotational degrees of freedom of the Cosserat continuum model.

With the use of force, bulk and surface balance and divergence theorem as known within average-field theory [4,7], the macroscopic average stresses are expressed as

$$
\begin{aligned}
\bar{\sigma}_{i j} & =\frac{1}{V} \int_{V} \sigma_{i j} d V=\frac{1}{V} \int_{V} \frac{\partial}{\partial x_{k}}\left(\sigma_{k j} x_{i}\right) d V=\frac{1}{V} \int_{S} n_{k} \sigma_{k j} x_{i} d S \\
& =\frac{1}{V} \int_{S} x_{i} t_{j} d S
\end{aligned}
$$

There are mainly two kinds of definitions for the average couple stress tensor expression in the literature. In the first version, the macroscopic average couple stresses are directly defined as the volume average of the corresponding microscopic couple stresses [22], i.e.

$\bar{\mu}_{i j}=\frac{1}{V} \int_{V} \mu_{i j} d V$

Based on this definition, Li et al. derived a version of Hill's lemma for Cosserat continuum in paper [27] as below

$$
\begin{aligned}
\overline{\sigma_{i j} \varepsilon_{i j}}-\bar{\sigma}_{i j} \bar{\varepsilon}_{i j}+\overline{\mu_{i j} \kappa_{i j}}-\bar{\mu}_{i j} \bar{\kappa}_{i j}= & \frac{1}{V} \int_{S}\left(u_{i}-\bar{u}_{i j} x_{j}\right)\left(n_{k} \sigma_{k i}\right. \\
& \left.-n_{k} \bar{\sigma}_{k i}\right) d S+\frac{1}{V} \int_{S}\left(\omega_{i}-\bar{\omega}_{i}\right) \\
& \times\left(n_{k} \mu_{k i}-n_{k} \bar{\mu}_{k i}\right) d S
\end{aligned}
$$

In the other version, in addition to the microscopic couple stresses, the effect of microscopic stresses is taken into account in the definition of average couple stresses [21,23], i.e.

$\bar{\mu}_{i j}=\frac{1}{V} \int_{V}\left(\mu_{i j}-x_{i} e_{j k l} \sigma_{k l}\right) d V$

Recently, Liu [28] presented a new version of Hill's lemma based on the definition (12) in the following form

$$
\begin{aligned}
\overline{\sigma_{i j} \varepsilon_{i j}}-\bar{\sigma}_{i j} \bar{\varepsilon}_{i j}+\overline{\mu_{i j} \kappa_{i j}}-\bar{\mu}_{i j} \bar{\kappa}_{i j}= & \frac{1}{V} \int_{S}\left(u_{i}-\bar{u}_{i, j} x_{j}\right)\left(n_{k} \sigma_{k i}\right. \\
& \left.-n_{k} \bar{\sigma}_{k i}\right) d S+\frac{1}{V} \int_{S}\left[\omega_{i}-\left(\bar{\omega}_{i}\right.\right. \\
& \left.\left.+\bar{\omega}_{i, j} x_{j}\right)\right]\left(n_{k} \mu_{k i}-n_{k} \bar{\mu}_{k i}\right) d S
\end{aligned}
$$

Analysis and discussion [28] also show that more versatile RVE boundary conditions in the strong form can be properly obtained from Hill's lemma (13) including:

(1) Homogeneous translational and rotational displacement boundary conditions

$$
\left.u_{i}\right|_{S}=\bar{u}_{i, j} x_{j},\left.\quad \omega_{i}\right|_{S}=\bar{\omega}_{i}+\bar{\omega}_{i, j} x_{j}
$$

(2) Mixed boundary conditions I, consisting of the translational displacement and the surface couple boundary condition

$\left.u_{i}\right|_{S}=\bar{u}_{i, j} x_{j},\left.\quad q_{i}\right|_{S}=\left.\left(n_{k} \mu_{k i}\right)\right|_{S}=n_{k} \bar{\mu}_{k i}$

(3) Mixed boundary conditions II, consisting of the surface traction and the rotational displacement boundary condition

$$
\left.t_{i}\right|_{S}=\left.\left(n_{k} \sigma_{k i}\right)\right|_{S}=n_{k} \bar{\sigma}_{k i},\left.\quad \omega_{i}\right|_{S}=\bar{\omega}_{i}+\bar{\omega}_{i, j} x_{j}
$$

These results motivate us to further develop the micro-macro homogenization scheme of granular materials based on the average-field theory of Cosserat continuum. The following sections will focus on the development of micro-macro homogenization formulation of granular materials based on Eqs. (12)-(16) as the extension of the work [28]. To make the formulation more concise and clear, some symbols used in the following sections are slightly different from those in paper [27].

\section{The microscopic discrete particle model of granular materials [27]}

The microscopic model in this paper follows the discrete particle model adopted in paper [27]. The primary idea and formulation are summarized and listed in this section.

It is usually assumed that the medium is composed of rigid discrete circular (spherical in 3D case) particles. Each particle possesses translational and rotational degrees of freedom. The movement of a typical particle follows the Newton's second law. The interaction between particles consists of both contact forces and contact moments, as shown in Fig. 3. According to the common assumption of discrete element method, as addressed in the contribution by Cundall and Strack [31], the particles are allowed to overlap one another at contact points. This overlapping behavior takes the place of the deformation of the individual particles. The magnitude of the overlap is related directly to the contact forces. However, these overlaps are small in relation to the particle sizes. The deformations of the individual particles are small in comparison with the deformation of a granular assembly as a whole. The latter deformation is due primarily to the movements of the particles as rigid bodies. Therefore, precise modeling of particle deformation is not necessary to obtain a good approximation of the mechanical behavior.

The particles within the RVE are partitioned into two parts, i.e. the interior and the peripheral. The particles classified into the latter part are assumed to contact with the material outside the RVE. The boundary $S$ of the RVE is actually an envelope of the peripheral particles of the assembly as shown in Fig. 2. There are only a finite number $N_{c}$ of material points located on the boundary $S$ of the RVE. We denote the total number of particles within the RVE with $N_{g}$ and numbers of the interior and the peripheral particles with $N_{i}$ and $N_{p}$ respectively, i.e. $N_{g}=N_{i}+N_{p}$. To consider a RVE in 2D case, the number of contacting points $N_{c}$ of the peripheral particles will be equal to $N_{p}$ or $N_{p}+4$, i.e. $N_{c}=N_{p}$ or $N_{c}=N_{p}+4$ for the RVE with a circular or rectangular boundary respectively. To simplify the discussion, we provisionally assume $N_{c}=N_{p}=N$ being a constant, i.e. each peripheral particle has only one contacting point with the boundary of the RVE, as shown in Figs. 2 and 4, and the total number $N$ of peripheral particles in the RVE is unchanged.

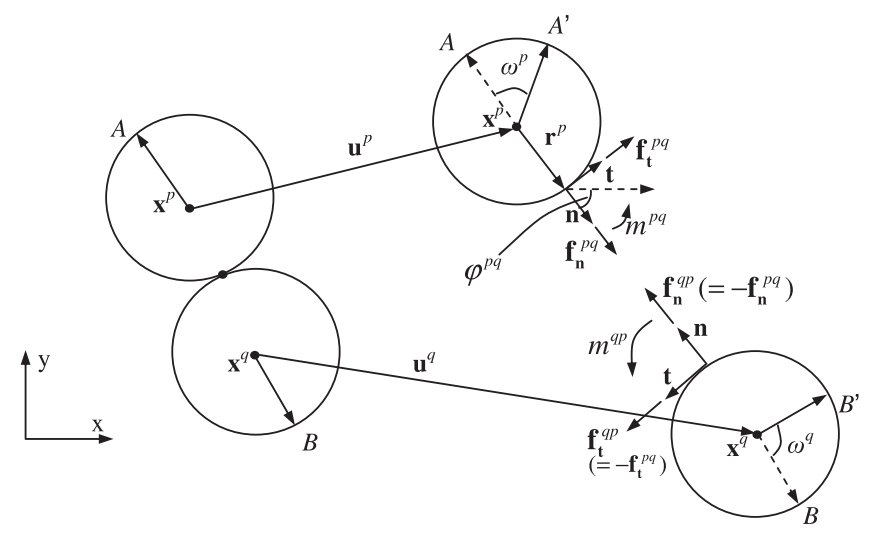

Fig. 3. Particles in contact. 


\subsection{Stiffness matrix relating two particles in contact}

The rates of the contacting force vector $\dot{\mathbf{f}}_{l}^{p q}$ applied to a particle $p$ by a neighbor particle $q$ via the contacting point can be expressed as $[10,31,32]$

$\dot{\mathbf{f}}_{l}^{p q}=\mathbf{K}_{l}^{p q} \dot{\mathbf{d}}^{p q}$

where $\dot{\mathbf{f}}_{l}^{p q}$ is referred to the local coordinate system defined at the contacting point as shown in Fig. 3 , and $\dot{\mathbf{d}}^{p q}$ is the rates of the displacement vector consisting of both translational and rotational displacements of the two typical particles and is referred to the global coordinate system. $\mathbf{K}_{l}^{p q}$ is the corresponding tangent stiffness matrix relating to the two typical particles and can be formulated according to the model used on the microscopic level to take into account physical and geometrical evolutions of the microstructures [33,34]. For the 2D case $\dot{\mathbf{f}}_{l}^{p q}$ and $\dot{\mathbf{d}}^{p q}$ can be expressed with their components as

$\dot{\mathbf{f}}_{l}^{p q}=\left[\begin{array}{lll}\dot{f}_{n}^{p q} & \dot{f}_{t}^{p q} & \dot{m}^{p q}\end{array}\right]^{\mathrm{T}}$

$\dot{\mathbf{d}}^{p q}=\left[\begin{array}{llllll}\dot{u}_{x}^{p} & \dot{u}_{y}^{p} & \dot{\omega}^{p} & \dot{u}_{x}^{q} & \dot{u}_{y}^{q} & \dot{\omega}^{q}\end{array}\right]^{\mathrm{T}}$

where subscripts $n, t$ denote the normal and tangential axes of the local coordinate system, respectively, and $x, y$ denote the global Cartesian coordinate axes. As the orientation of the local coordinates $n-t$ with respect to the global coordinates $x-y$ is denoted by the angle $\varphi^{p q}$, as shown in Fig. 3, the rates of the contacting force vector $\mathbf{f}^{p q}$ applied at the center of the particle $p$ by the particle $q$ referring to the global coordinates are given by

$\dot{\mathbf{f}}^{p q}=\mathbf{T}^{p q} \dot{\mathbf{f}}_{l}^{p q}$

in which the transformation matrix has the form

$\mathbf{T}^{p q}=\left[\begin{array}{ccc}\cos \varphi^{p q} & -\sin \varphi^{p q} & 0 \\ \sin \varphi^{p q} & \cos \varphi^{p q} & 0 \\ 0 & r^{p} & 1\end{array}\right]$

where $r^{p}$ is the radius of the circular particle $p$. Substitution of Eq. (17) into Eq. (20) gives

$\dot{\mathbf{f}}^{p q}=\mathbf{T}^{p q} \mathbf{K}_{l}^{p q} \dot{\mathbf{d}}^{p q}=\mathbf{K}^{p q} \dot{\mathbf{d}}^{p q}$

where $\mathbf{K}^{p q}$ is the stiffness matrix referred to the global coordinates. The sum of the rate contacting forces $\dot{\mathbf{f}}^{p q}$ applied at the center of a typical particle $p$ by all of the neighboring particles in contacts with the particle $p$ is given by

$\dot{\mathbf{f}}^{p}=\sum_{q=1}^{N_{q}} \dot{\mathbf{f}}^{p q}=\sum_{q=1}^{N_{q}} \mathbf{K}^{p q} \dot{\mathbf{d}}^{p q}$

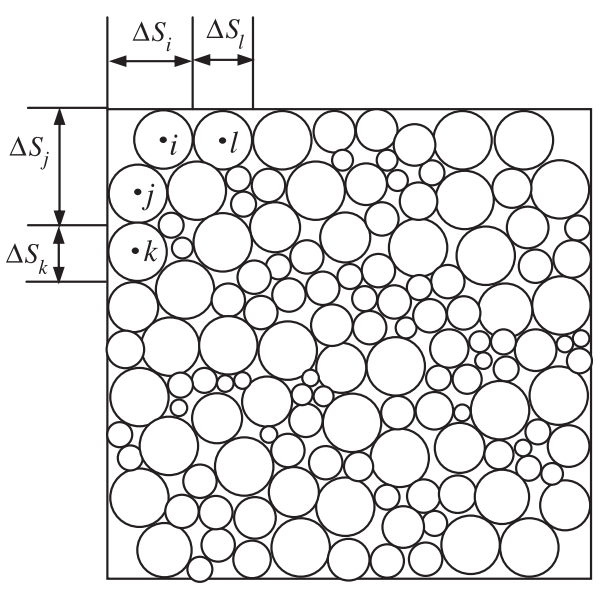

Fig. 4. The microstructure of granular RVE.

\subsection{Stiffness equation for the discrete particle assembly}

We denote the displacement vector $\mathbf{d}$ of the discrete particle assembly of the RVE with

$\mathbf{d}=\left\{\begin{array}{l}\mathbf{d}^{I} \\ \mathbf{d}^{B}\end{array}\right\} \quad$ with $\quad \mathbf{d}^{I}=\left\{\begin{array}{c}\mathbf{u}^{I} \\ \omega^{I}\end{array}\right\} \quad$ and $\quad \mathbf{d}^{B}=\left\{\begin{array}{c}\mathbf{u}^{B} \\ \omega^{B}\end{array}\right\}$

where $\mathbf{u}$ and $\omega$ denote the translational and angular displacement vector respectively, the superscripts $I$ and $B$ stand for the interior and the boundary (peripheral) degrees of freedom, i.e. $\mathbf{d}^{I}$ and $\mathbf{d}^{B}$ list the displacements of the centers of all interior and peripheral particles respectively. The rates of the stiffness equation of the discrete assembly RVE can be expressed by

$\left[\begin{array}{ll}\mathbf{K}_{u u}^{b} & \mathbf{K}_{u \omega}^{b} \\ \mathbf{K}_{\omega u}^{b} & \mathbf{K}_{\omega \omega}^{b}\end{array}\right]\left\{\begin{array}{l}\dot{\mathbf{u}}^{B} \\ \dot{\boldsymbol{\omega}}^{B}\end{array}\right\}=\left\{\begin{array}{l}\dot{\mathbf{f}}^{B, e x t} \\ \dot{\mathbf{m}}^{B, e x t}\end{array}\right\}$

in which $\dot{\mathbf{f}}^{\text {Bext }}$ and $\dot{\mathbf{m}}^{\text {Bext }}$ list the external forces and moments directly exerted on the centers of the peripheral particles, $\mathbf{K}_{u u}^{b}, \mathbf{K}_{u \omega}^{b}, \mathbf{K}_{\omega u}^{b}$ and $\mathbf{K}_{\omega \omega}^{b}$ are the block stiffness matrices.

\subsection{Transformation of the reference point of the RVE stiffness equation}

In the discrete element model, the displacement degrees of freedom and the corresponding forces are usually defined at the particle centers, as expressed by Eq. (25). In the micro-macro homogenization, the microscopic discrete particle assembly contacts with the material outside the RVE through the peripheral points, as shown in Figs. 2 and 4. The imposition of RVE boundary conditions and the definition of effective continuum quantities are referred to peripheral contacting points. Therefore, the RVE governing Eq. (25) should be further transformed.

According to the kinematics and statics of rigid circular particle, we have

$\dot{\boldsymbol{\omega}}^{B}=\dot{\boldsymbol{\omega}}^{C}, \quad \dot{\mathbf{u}}^{B}=\dot{\mathbf{u}}^{C}-\mathbf{R} \dot{\boldsymbol{\omega}}^{C}$

$\dot{\mathbf{f}}^{B, e x t}=\dot{\mathbf{f}}^{C}, \quad \dot{\mathbf{m}}^{B, e x t}=\dot{\mathbf{m}}^{C}+\mathbf{R}^{T} \dot{\mathbf{f}}^{C}$

in which $\dot{\mathbf{u}}^{C}$ and $\dot{\omega}^{C}$ list the translational and rotational displacement rates of the peripheral contacting points, $\dot{\mathbf{f}}^{C}$ and $\dot{\mathbf{m}}^{C}$ list the external forces and moments rates exerted on the peripheral contacting points,

$$
\begin{aligned}
\mathbf{R} & =\left[\begin{array}{cccccc}
\mathbf{R}^{1} & \mathbf{0} & \mathbf{0} & \mathbf{0} & \mathbf{0} & \mathbf{0} \\
\mathbf{0} & . & \mathbf{0} & \mathbf{0} & \mathbf{0} & \mathbf{0} \\
\mathbf{0} & \mathbf{0} & \mathbf{R}^{c} & \mathbf{0} & \mathbf{0} & \mathbf{0} \\
\mathbf{0} & \mathbf{0} & \mathbf{0} & . & \mathbf{0} & \mathbf{0} \\
\mathbf{0} & \mathbf{0} & \mathbf{0} & \mathbf{0} & . & \mathbf{0} \\
\mathbf{0} & \mathbf{0} & \mathbf{0} & \mathbf{0} & \mathbf{0} & \mathbf{R}^{N}
\end{array}\right] \\
\mathbf{R}^{c} & =\left[\begin{array}{lll}
-r_{2}^{c} & r_{1}^{c}
\end{array}\right]^{T}
\end{aligned}
$$

Substitution of Eqs. (26) and (27) into Eq. (25) leads to

$\dot{\mathbf{f}}^{C}=\mathbf{K}^{f u} \dot{\mathbf{u}}^{C}+\mathbf{K}^{f \omega} \dot{\boldsymbol{\omega}}^{C}$

$\dot{\mathbf{m}}^{C}=\mathbf{K}^{m u} \dot{\mathbf{u}}^{C}+\mathbf{K}^{m \omega} \dot{\boldsymbol{\omega}}^{C}$

where

$$
\begin{aligned}
& \mathbf{K}^{f u}=\mathbf{K}_{u u}^{b} \\
& \mathbf{K}^{f \omega}=\left(\mathbf{K}_{u \omega}^{b}-\mathbf{K}_{u u}^{b} \mathbf{R}\right) \\
& \mathbf{K}^{m u}=\mathbf{K}_{\omega u}^{b}-\mathbf{R}^{\mathrm{T}} \mathbf{K}_{u u}^{b} \\
& \mathbf{K}^{m \omega}=\mathbf{K}_{\omega \omega}^{b}-\mathbf{R}^{\mathrm{T}} \mathbf{K}_{u \omega}^{b}-\mathbf{K}_{\omega u}^{b} \mathbf{R}+\mathbf{R}^{\mathrm{T}} \mathbf{K}_{u u}^{b} \mathbf{R}
\end{aligned}
$$


Eqs. (30) and (31) give the external force rates $\dot{\mathbf{f}}^{C}$ and the external moment rates $\dot{\mathbf{m}}^{C}$ applied at peripheral contacting points of the RVE in terms of $\dot{\mathbf{u}}^{C}$ and $\dot{\boldsymbol{\omega}}^{C}$, i.e. the translational and rotational displacement rates imposed to the contacting points.

\section{The macroscopic stress and couple stress}

To determine the macroscopic average stresses and the average couple stresses in the homogenization procedure of granular materials in the frame of the average-field theory, the integrals of Eqs. (9) and (12) should be expressed in terms of the discrete quantities at the peripheral contacting points of the discrete particle assembly RVE. The boundary integrals given by Eq. (9) can be discretized as

$\overline{\boldsymbol{\sigma}}=\frac{1}{V} \int_{S} \mathbf{x} \otimes \mathbf{t} d S=\frac{1}{V} \sum_{c=1}^{N} \mathbf{x}^{c} \otimes \mathbf{t}^{c} \Delta S^{c}=\frac{1}{V} \sum_{c=1}^{N} \mathbf{x}^{c} \otimes \mathbf{f}^{c}$

With Eqs. (3)-(6), we have

$$
\begin{aligned}
\int_{V}\left(\mu_{i j}+e_{j k l} \sigma_{i l} x_{k}\right) d V & =\int_{V} \frac{\partial}{\partial x_{m}}\left[x_{i}\left(\mu_{m j}+e_{j k l} x_{k} \sigma_{m l}\right)\right] d V \\
& =\int_{S} x_{i} n_{m}\left(\mu_{m j}+e_{j k l} x_{k} \sigma_{m l}\right) d S \\
& =\int_{S}\left(x_{i} q_{j}+e_{j k l} x_{i} x_{k} t_{l}\right) d S
\end{aligned}
$$

On the other hand,

$$
\begin{aligned}
\int_{V}\left(\mu_{i j}+e_{j k l} \sigma_{i l} x_{k}\right) d V= & \int_{V}\left(\mu_{i j}+e_{j k l} \sigma_{i l} x_{k}\right) d V-\int_{V} x_{i} e_{j k l} \sigma_{k l} d V \\
& +\int_{V} x_{i} e_{j k l} \sigma_{k l} d V \\
= & \int_{V}\left(\mu_{i j}-x_{i} e_{j k l} \sigma_{k l}\right) d V+e_{j k l} \int_{V}\left(x_{i} \sigma_{k l}+x_{k} \sigma_{i l}\right) d V \\
= & \int_{V}\left(\mu_{i j}-x_{i} e_{j k l} \sigma_{k l}\right) d V+e_{j k l} \int_{V} \frac{\partial}{\partial x_{m}}\left(x_{i} x_{k} \sigma_{m l}\right) d V \\
= & \int_{V}\left(\mu_{i j}-x_{i} e_{j k l} \sigma_{k l}\right) d V+e_{j k l} \int_{S} x_{i} x_{k} t_{l} d S
\end{aligned}
$$

From Eqs. (34) and (35), we can obtain

$$
\begin{aligned}
\frac{1}{V} \int_{V}\left(\mu_{i j}-x_{i} e_{j k l} \sigma_{k l}\right) d V= & \int_{S}\left(x_{i} q_{j}+e_{j k l} x_{i} x_{k} t_{l}\right) d S \\
& -\int_{V} e_{j k l} x_{i} x_{k} t_{l} d V \\
& =\int_{S} x_{i} q_{j} d S
\end{aligned}
$$

Thus the macroscopic couple stress tensor of Eq. (12) can be expressed as

$\overline{\boldsymbol{\mu}}=\frac{1}{V} \int_{S} \mathbf{x} \otimes \mathbf{q} d S=\frac{1}{V} \sum_{c=1}^{N} \mathbf{x}^{c} \otimes \mathbf{q}^{c} \Delta S^{c}=\frac{1}{V} \sum_{c=1}^{N} \mathbf{x}^{c} \otimes \mathbf{m}^{c}$

The static relation between a discrete particle assembly and its effective Cosserat continuum model has been discussed by some authors $[10,13,14,35-37]$. The definition of effective stress tensor has arrived at a uniform expression identical with Eq. (33), which can be further expressed in terms of the contact quantities over the RVE domain as below

$\bar{\sigma}_{i j}=\frac{1}{V} \sum_{c \in B} x_{i}^{c} f_{j}^{c}=\frac{1}{V} \sum_{c \in V \cup B} l_{i}^{c} f_{j}^{c}$

in which $f_{j}^{c}$ is the contact force vector between two particles, $c \in B$ denote the set of peripheral contacting points of the RVE, $c \in V U B$ denote the set of both the internal $(V)$ and peripheral $(B)$ contacting points over the RVE. $l_{i}^{c}=x_{i}^{q}-x_{i}^{p}$ is the so-called branch vector connecting the centers of two contacting particles $p$ and $q$.
The definition of effective couple stress tensor of a discrete particle assembly is still open and does not arrive at a unified conclusion. Some authors give definition and derivation based on a typical particle and its neighborhood. The representative work includes the contribution by Suiker et al. [35] and Tordesillas and Walsh [13]. Others hold that the stress measures should be defined on the basis of a statistically homogeneous RVE containing a sufficient number of particles so as to preserve the essential characteristics of the equivalent continuum modeling. This is consistent with the basic assumptions of the average-field theory. The representative work of this type includes the contribution by Kruyt [37] and Chang and Kuhn [14]. Kruyt gives definition and derivation based on the scale separation assumption and moments of equilibrium equations of the discrete particle assembly. Chang and Kuhn apply the principle of virtual work to the discrete system and revealed the equivalence of expressions summing over the set $c \in B$ and over the set of $c \in V U B$.

In the previous work of the average-field theory for Cosserat continuum [27], the macro average couple stress tensor is defined as Eq. (10), i.e. $\bar{\mu}_{i j}=\frac{1}{V} \int_{V} \mu_{i j} d V$. According to the derivation in paper [37], $\bar{\mu}_{i j}$ can be further expressed in terms of the contact quantities over the RVE domain as below

$\bar{\mu}_{i j}=\frac{1}{V} \int_{V} \mu_{i j} d V=\frac{1}{V} \sum_{c \in V \cup B} l_{i}^{c} m_{j}^{c}$

in which $m_{j}^{c}$ is the contact moment vector between two particles.

According to the results from the work by Chang and Kuhn [14], Eq. (37) can be equivalently expressed in terms of the contact quantities over the RVE domain in the form

$\bar{\mu}_{i j}=\frac{1}{V} \sum_{c \in B} x_{i}^{c} m_{j}^{c}=\frac{1}{V} \sum_{c \in V \cup B}\left[l_{i}^{c} m_{j}^{c}+e_{j k} f_{k}^{c}\left(J_{l i}^{c}-x_{l}^{c} l_{i}^{c}\right)\right]$

in which

$l_{i}^{c}=\left\{\begin{array}{ll}x_{i}^{q}-x_{i}^{p} & c \in V \\ x_{i}^{c}-x_{i}^{p} & c \in B\end{array} ; J_{i j}^{c}= \begin{cases}x_{i}^{q} x_{j}^{q}-x_{i}^{p} x_{j}^{p} & c \in V \\ x_{i}^{c} x_{j}^{c}-x_{i}^{p} x_{j}^{p} & c \in B\end{cases}\right.$

Thus, a new definition of the effective couple stress tensor Eq. (40) is given for the Cosserat continuum modeling of a discrete particle assembly in light of Hill's lemma (13) combining with the contribution [14]. The first equation in (40), i.e. $\bar{\mu}_{i j}=\frac{1}{V} \sum_{c \in B} x_{i}^{c} m_{j}^{c}$, is strictly derived in light of the average-field theory, as shown in Eqs. (34)(37). The second equation in (40), i.e. $\frac{1}{V} \sum_{c \in B} x_{i}^{c} m_{j}^{c}=\frac{1}{V} \sum_{c \in V \cup B}$ $\left[l_{i}^{c} m_{j}^{c}+e_{j k k} f_{k}^{c}\left(J_{l i}^{c}-x_{l}^{c} l_{i}^{c}\right)\right]$, is one of the essential results from paper [14], which insures that a shifting of the coordinate system will not affect the expression of the effective couple stress tensor.

It should be again stressed that the derivation of Eq. (40) is based on the average-field theory which is essentially different from the work based on a typical particle and its neighborhood $[13,35]$. On the other hand, Eq. (40) is also slightly different from the macro couple stress $\bar{\mu}_{i j}^{0}=\frac{1}{V} \sum_{c \in V \cup B}\left[l_{i}^{c} m_{j}^{c}+e_{j k} f_{k}^{c}\left(\frac{1}{2} J_{l i}^{c}-x_{l}^{c} l_{i}^{c}\right)\right]$ defined by Chang and Kuhn [14]. The origin of this difference lies in that Chang and Kuhn assumed a gradient-enhanced Cosserat continuum model in which second-order gradients of translational displacements $u_{i, j k}$ is considered. While the standard (first-order) Cosserat continuum model is adopted in our presentation.

Furthermore, the above formulations inspire us to reconsider the issue of effective couple stress tensor definition of a discrete particle assembly from the view of the average-field theory, i.e. the definition of effective couple stress tensor is not necessarily unique and the two definitions (39) and (40) can both be properly used in the micro-macro homogenization of granular materials, provided that corresponding kinematical quantities are properly chosen and determined in light of Hill's lemma (11) and/or (13). 
Therefore, it provides an alternative theoretical reference for the definition and use of effective couple stress tensor of granular materials with the premise that micro-macro energy equivalence is satisfied in the homogenization approach.

\section{Micromechanically based constitutive models consistent with RVE boundary conditions}

In the homogenization approach based on the average-field theory in paper [27], the micromechanically based constitutive relation is consistent with RVE boundary conditions. In other words, different types of RVE boundary conditions will lead to constitutive models with different forms. In this section, the rate forms of micromechanically based constitutive models for granular materials are derived according to RVE boundary conditions given by Eqs. (14)-(16).

\subsection{Constitutive model consistent with homogeneous translational} and rotational displacement boundary conditions

As the homogeneous displacement boundary conditions $\left.u_{i}\right|_{S}=\bar{u}_{i, j} x_{j},\left.\omega_{i}\right|_{S}=\bar{\omega}_{i}+\bar{\omega}_{i, j} x_{j}$ are imposed on the continuous boundary $S$ of the Cosserat continuum equivalent RVE to ensure the satisfaction of the Hill-Mandel condition, the discrete counterpart of the boundary conditions prescribed on the discrete $N$ contacting points of the peripheral particles are written for a typical contacting point $c$ in the rate form

$\dot{u}_{i}^{c}=\dot{\bar{u}}_{i, j} x_{j}^{c} \quad$ or $\quad \dot{\mathbf{u}}^{c}=\mathbf{x}^{c} \cdot \dot{\mathbf{E}}$

$\dot{\omega}_{i}^{c}=\dot{\bar{\omega}}_{i}+\dot{\bar{\omega}}_{i, j} x_{j}^{c} \quad$ or $\quad \dot{\boldsymbol{\omega}}^{c}=\dot{\overline{\boldsymbol{\omega}}}+\mathbf{x}^{c} \cdot \dot{\overline{\boldsymbol{\kappa}}}$

where $\overline{\mathbf{E}}=\bar{E}_{j i}=\bar{u}_{i, j}$ denote the gradient of the macroscopic displacements.

According to Eqs. (33) and (37), the macroscopic stress and couple stress rates can be expressed in terms of the microscopic quantities of the discrete particle assembly as below

$\dot{\overline{\boldsymbol{\sigma}}}=d\left(\frac{1}{V}\right) \sum_{c=1}^{N} \mathbf{x}^{c} \otimes \mathbf{f}^{c}+\frac{1}{V} \sum_{c=1}^{N} \mathbf{x}^{c} \otimes \dot{\mathbf{f}}^{c}+\frac{1}{V} \sum_{c=1}^{N} \dot{\mathbf{x}}^{c} \otimes \mathbf{f}^{c}$

$\dot{\overline{\boldsymbol{\mu}}}=d\left(\frac{1}{V}\right) \sum_{c=1}^{N} \mathbf{X}^{c} \otimes \mathbf{m}^{c}+\frac{1}{V} \sum_{c=1}^{N} \mathbf{x}^{c} \otimes \dot{\mathbf{m}}^{c}+\frac{1}{V} \sum_{c=1}^{N} \dot{\mathbf{x}}^{c} \otimes \mathbf{m}^{c}$

It is observed from Eqs. (44) and (45) that the averaged stress and couple stress rates depend on not only the changes of the contacting forces and moments assigned to the discrete peripheral particles, but also the evolutions of geometric configuration characterized by the changes of the positions of those particles and the volume of the RVE.

According to the definition given in Section $4.3, \dot{\mathbf{f}}^{C}$ is the external force vector composed of all the external forces applied to the contacting point of the peripheral particles. The rate of translational contacting force $\dot{\mathbf{f}}^{c}$, which is required to be further determined in Eq. (44), is applied at the contacting point of the $c^{\prime}$ th peripheral particle of the RVE. Eq. (29) can be expanded as

$$
\begin{aligned}
& \left\{\begin{array}{c}
\dot{\mathbf{f}}^{1} \\
\cdot \\
\dot{\mathbf{f}^{c}} \\
\cdot \\
\dot{\mathbf{f}^{N}}
\end{array}\right\}=\left[\begin{array}{ccccc}
\mathbf{K}^{f u(11)} & \cdot & \mathbf{K}^{f u(1 c)} & \cdot & \mathbf{K}^{f u(1 N)} \\
\cdot & \cdot & \cdot & \cdot & \cdot \\
\mathbf{K}^{f u(c 1)} & \cdot & \mathbf{K}^{f u(c c)} & \cdot & \mathbf{K}^{f u(c N)} \\
\cdot & \cdot & \cdot & \cdot & \cdot \\
\mathbf{K}^{f u(N 1)} & \cdot & \mathbf{K}^{f u(N c)} & \cdot & \mathbf{K}^{f u(N N)}
\end{array}\right]\left\{\begin{array}{l}
\dot{\mathbf{u}}^{1} \\
\cdot \\
\dot{\mathbf{u}}^{c} \\
\cdot \\
\dot{\mathbf{u}}^{N}
\end{array}\right\} \\
& +\left[\begin{array}{ccccc}
\mathbf{K}^{f \omega(11)} & \cdot & \mathbf{K}^{f \omega(1 c)} & \cdot & \mathbf{K}^{f \omega(1 N)} \\
\cdot & \cdot & \cdot & \cdot & \cdot \\
\mathbf{K}^{f \omega(c 1)} & \cdot & \mathbf{K}^{f \omega(c c)} & \cdot & \mathbf{K}^{f \omega(c N)} \\
\cdot & \cdot & \cdot & \cdot & \cdot \\
\mathbf{K}^{f \omega(N 1)} & \cdot & \mathbf{K}^{f \omega(N c)} & \cdot & \mathbf{K}^{f \omega(N 1)}
\end{array}\right]\left\{\begin{array}{c}
\dot{\omega}^{1} \\
\cdot \\
\dot{\omega}^{c} \\
\cdot \\
\dot{\omega}^{N}
\end{array}\right\}
\end{aligned}
$$

From Eq. (46), $\dot{\mathbf{f}}^{c}$ can be expressed as

$\dot{\mathbf{f}}^{c}=\sum_{b=1}^{N}\left[\mathbf{K}^{f u(c b)} \dot{\mathbf{u}}^{b}+\mathbf{K}^{f \omega(c b)} \dot{\boldsymbol{\omega}}^{b}\right]$

Similarly, $\dot{\mathbf{m}}^{c}$ is expressed as

$\dot{\mathbf{m}}^{c}=\sum_{b=1}^{N}\left[\mathbf{K}^{m u(c b)} \dot{\mathbf{u}}^{b}+\mathbf{K}^{m \omega(c b)} \dot{\boldsymbol{\omega}}^{b}\right]$

To establish the micromechanically based macroscopic constitutive model, the rate form of the macroscopic stress-strain relations and corresponding consistent tangent modular tensors are required to be derived. According to the boundary conditions described by Eqs. (42) and (43), the macroscopic kinematical quantities $\dot{\mathbf{E}}, \dot{\overline{\boldsymbol{\omega}}}$, $\dot{\overline{\boldsymbol{\kappa}}}$ will act as "external loads" to apply to the RVE. The micromechanical analysis for the RVE modeled as a discrete particle assembly is then performed and the micromechanically based macroscopic consistent tangent modular tensors are derived.

After a series of derivation as shown in Appendices A.1,A.2,A.3, the three terms at the right hand side of Eq. (44) can be further deduced to result in the following expressions:

$d\left(\frac{1}{V}\right) \sum_{c=1}^{N} \mathbf{x}^{c} \otimes \mathbf{f}^{c}=-(\overline{\boldsymbol{\sigma}} \otimes \mathbf{I}): \dot{\overline{\mathbf{E}}}$

$\frac{1}{V} \sum_{c=1}^{N} \mathbf{X}^{c} \otimes \dot{\mathbf{f}}^{c}=\mathbf{D}^{u, \sigma E-1}: \dot{\overline{\mathbf{E}}}+\mathbf{D}^{u, \sigma \omega} \cdot \dot{\overline{\boldsymbol{\omega}}}+\mathbf{D}^{u, \sigma \kappa}: \dot{\overline{\boldsymbol{\kappa}}}$

$\frac{1}{V} \sum_{c=1}^{N} \dot{\mathbf{x}}^{c} \otimes \mathbf{f}^{c}=\mathbf{D}^{u, \sigma E-2}: \dot{\overline{\mathbf{E}}}$

with the modular tensors given below

$\mathbf{D}^{u, \sigma E-1}=\frac{1}{V} \sum_{c=1}^{N} \sum_{b=1}^{N} x_{i}^{c} K_{j l}^{f u(c b)} x_{k}^{b} \mathbf{e}_{i} \otimes \mathbf{e}_{j} \otimes \mathbf{e}_{k} \otimes \mathbf{e}_{l}$
$\mathbf{D}^{u, \sigma \omega}=\frac{1}{V} \sum_{c=1}^{N} \sum_{b=1}^{N} x_{i}^{c} K_{j k}^{f(c(c)} \mathbf{e}_{i} \otimes \mathbf{e}_{j} \otimes \mathbf{e}_{k}$
$\mathbf{D}^{u, \sigma \kappa}=\frac{1}{V} \sum_{c=1}^{N} \sum_{b=1}^{N} x_{i}^{c} K_{j l}^{f \omega(c b)} x_{k}^{b} \mathbf{e}_{i} \otimes \mathbf{e}_{j} \otimes \mathbf{e}_{k} \otimes \mathbf{e}_{l}$
$\mathbf{D}^{u, \sigma E-2}=\frac{1}{V} \sum_{c=1}^{N} \delta_{i l} x_{k}^{c} f_{j}^{c} \mathbf{e}_{i} \otimes \mathbf{e}_{j} \otimes \mathbf{e}_{k} \otimes \mathbf{e}_{l}$

Substitution of Eqs. (49)-(51) into Eq. (44) results in

$$
\begin{gathered}
\dot{\overline{\boldsymbol{\sigma}}}=-(\overline{\boldsymbol{\sigma}} \otimes \mathbf{I}): \dot{\overline{\mathbf{E}}}+\mathbf{D}^{u, \sigma E-1}: \dot{\overline{\mathbf{E}}}+\mathbf{D}^{u, \sigma \omega} \cdot \dot{\overline{\boldsymbol{\omega}}}+\mathbf{D}^{u, \sigma \kappa}: \dot{\overline{\boldsymbol{\kappa}}}+\mathbf{D}^{u, \sigma E-2} \\
: \dot{\overline{\mathbf{E}}}=\mathbf{D}^{u, \sigma E}: \dot{\overline{\mathbf{E}}}+\mathbf{D}^{u, \sigma \omega} \cdot \dot{\overline{\boldsymbol{\omega}}}+\mathbf{D}^{u, \sigma \kappa}: \dot{\overline{\boldsymbol{\kappa}}}
\end{gathered}
$$

in which

$\mathbf{D}^{u, \sigma E}=-(\overline{\boldsymbol{\sigma}} \otimes \mathbf{I})+\mathbf{D}^{u, \sigma E-1}+\mathbf{D}^{u, \sigma E-2}$

The three terms at the right hand side of Eq. (45) can be further deduced to result in the following expressions, as shown in Appendices A.4,A.5,A.6

$d\left(\frac{1}{V}\right) \sum_{c=1}^{N} \mathbf{x}^{c} \otimes \mathbf{m}^{c}=-(\overline{\boldsymbol{\mu}} \otimes \mathbf{I}): \dot{\overline{\mathbf{E}}}$

$\frac{1}{V} \sum_{c=1}^{N} \mathbf{x}^{c} \otimes \dot{\mathbf{m}}^{c}=\mathbf{D}^{u, \mu E-1}: \dot{\overline{\mathbf{E}}}+\mathbf{D}^{u, \mu \omega} \cdot \dot{\overline{\boldsymbol{\omega}}}+\mathbf{D}^{u, \mu \kappa}: \dot{\bar{\kappa}}$

$\frac{1}{V} \sum_{c=1}^{N} \dot{\mathbf{x}}^{c} \otimes \mathbf{m}^{c}=\mathbf{D}^{u, \mu E-2}: \dot{\overline{\mathbf{E}}}$

The modular tensors in the above three equations are given as below 
$\mathbf{D}^{u, \mu E-1}=\frac{1}{V} \sum_{c=1}^{N} \sum_{b=1}^{N} x_{i}^{c} K_{j l}^{m u(c b)} x_{k}^{b} \mathbf{e}_{i} \otimes \mathbf{e}_{j} \otimes \mathbf{e}_{k} \otimes \mathbf{e}_{l}$

$\mathbf{D}^{u, \mu \omega}=\frac{1}{V} \sum_{c=1}^{N} \sum_{b=1}^{N} X_{i}^{c} K_{j k}^{m \omega(c b)} \mathbf{e}_{i} \otimes \mathbf{e}_{j} \otimes \mathbf{e}_{k}$

$\mathbf{D}^{u, \mu \kappa}=\frac{1}{V} \sum_{c=1}^{N} \sum_{b=1}^{N} x_{i}^{c} K_{j l}^{m \omega(c b)} x_{k}^{b} \mathbf{e}_{i} \otimes \mathbf{e}_{j} \otimes \mathbf{e}_{k} \otimes \mathbf{e}_{l}$

$\mathbf{D}^{u, \mu E-2}=\frac{1}{V} \sum_{c=1}^{N} \delta_{i l} x_{k}^{c} m_{j}^{c} \mathbf{e}_{i} \otimes \mathbf{e}_{j} \otimes \mathbf{e}_{k} \otimes \mathbf{e}_{l}$

Substitution of Eqs. (58)-(60) into Eq. (45) results in

$$
\begin{aligned}
\dot{\overline{\boldsymbol{\mu}}} & =-(\overline{\boldsymbol{\mu}} \otimes \mathbf{I}): \dot{\overline{\mathbf{E}}}+\mathbf{D}^{u, \mu E-1}: \dot{\overline{\mathbf{E}}}+\mathbf{D}^{u, \mu \omega} \cdot \dot{\overline{\boldsymbol{\omega}}}+\mathbf{D}^{u, \mu \kappa}: \dot{\overline{\boldsymbol{\kappa}}}+\mathbf{D}^{u, \mu E-2} \\
& : \dot{\overline{\mathbf{E}}}=\mathbf{D}^{u, \mu E}: \dot{\overline{\mathbf{E}}}+\mathbf{D}^{u, \mu \omega} \cdot \dot{\overline{\boldsymbol{\omega}}}+\mathbf{D}^{u, \mu \kappa}: \dot{\overline{\boldsymbol{\kappa}}}
\end{aligned}
$$

in which

$\mathbf{D}^{u, \mu E}=-(\overline{\boldsymbol{\mu}} \otimes \mathbf{I})+\mathbf{D}^{u, \mu E-1}+\mathbf{D}^{u, \mu E-2}$

In summary, micromechanically based macroscopic constitutive relations consistent with the homogeneous translational and rotational displacement boundary conditions can be constituted with Eqs. (56) and (65), and re-expressed together as below

$\dot{\overline{\boldsymbol{\sigma}}}=\mathbf{D}^{u, \sigma E}: \dot{\overline{\mathbf{E}}}+\mathbf{D}^{u, \sigma \omega} \cdot \dot{\overline{\boldsymbol{\omega}}}+\mathbf{D}^{u, \sigma \kappa}: \dot{\overline{\boldsymbol{\kappa}}}$

$\dot{\overline{\boldsymbol{\mu}}}=\mathbf{D}^{u, \mu E}: \dot{\overline{\mathbf{E}}}+\mathbf{D}^{u, \mu \omega} \cdot \dot{\overline{\boldsymbol{\omega}}}+\mathbf{D}^{u, \mu \kappa}: \dot{\overline{\boldsymbol{\kappa}}}$

\subsection{Constitutive model consistent with the mixed RVE boundary} conditions I

As the mixed boundary conditions I, i.e. $\left.u_{i}\right|_{S}=\bar{u}_{i, j} x_{j},\left.q_{i}\right|_{S}$ $=\left.\left(n_{k} \mu_{k i}\right)\right|_{s}=n_{k} \bar{\mu}_{k i}$ are imposed on the RVE boundary to ensure the satisfaction of Hill-Mandel condition, the counterpart with rate forms prescribed on the discrete $N$ contacting points of the peripheral particles are written for a typical contacting point $c$ as

$\dot{\mathbf{u}}^{c}=\mathbf{x}^{c} \cdot \dot{\overline{\mathbf{E}}}$

$\dot{\mathbf{m}}^{c}=\mathbf{n}^{c} \cdot \dot{\overline{\boldsymbol{\mu}}} \Delta S^{c}$

The macroscopic stress rates have been given by Eq. (44). According to Eq. (8), the macroscopic curvature rates can be expressed as

$$
\begin{aligned}
\dot{\bar{\kappa}}= & d\left(\frac{1}{V}\right) \sum_{c=1}^{N} \mathbf{n}^{c} \otimes \omega^{c} \Delta S^{c}+\frac{1}{V} \sum_{c=1}^{N} \mathbf{n}^{c} \otimes \dot{\omega}^{c} \Delta S^{c} \\
& +\frac{1}{V} \sum_{c=1}^{N} \dot{\mathbf{n}}^{c} \otimes \omega^{c} \Delta S^{c}
\end{aligned}
$$

in which $\Delta S^{c}$ is the area weight of the peripheral particle $c$ as shown in Figs. 2 and 4.

Eqs. (29) and (30) can be equivalently transformed in the following form

$\dot{\mathbf{f}}^{C}=\mathbf{K}^{\mathrm{Ifu}} \dot{\mathbf{u}}^{C}+\mathbf{K}^{\mathrm{Ifm}} \dot{\mathbf{m}}^{\mathrm{C}}$

$\dot{\omega}^{C}=\mathbf{K}^{I \omega u} \dot{\mathbf{u}}^{C}+\mathbf{K}^{I \omega m} \dot{\mathbf{m}}^{\mathrm{C}}$

in which

$$
\begin{aligned}
& \mathbf{K}^{\mathrm{Ifu}}=\mathbf{K}^{f u}-\mathbf{K}^{f \omega}\left[\mathbf{K}^{m \omega}\right]^{-1} \mathbf{K}^{m u} \\
& \mathbf{K}^{\mathrm{Ifm}}=\mathbf{K}^{f \omega}\left[\mathbf{K}^{m \omega}\right]^{-1} \\
& \mathbf{K}^{\mathrm{I} \omega u}=-\left[\mathbf{K}^{m \omega}\right]^{-1} \mathbf{K}^{m u} \\
& \mathbf{K}^{\mathrm{I} \omega m}=\left[\mathbf{K}^{m \omega}\right]^{-1}
\end{aligned}
$$

Thus we have
$\dot{\mathbf{f}}^{c}=\sum_{b=1}^{N}\left[\mathbf{K}^{\mathrm{I} f u(c b)} \dot{\mathbf{u}}^{b}+\mathbf{K}^{\mathrm{Ifm(cb)}} \dot{\mathbf{m}}^{b}\right]$

$\dot{\omega}^{c}=\sum_{b=1}^{N}\left[\mathbf{K}^{I \omega u(c b)} \dot{\mathbf{u}}^{b}+\mathbf{K}^{I \omega m(c b)} \dot{\mathbf{m}}^{b}\right]$

According to the derivations in Appendices B.1 and B.2, Eq. (44) can be further expressed under the mixed RVE boundary conditions I in the following form

$$
\begin{aligned}
\dot{\overline{\boldsymbol{\sigma}}} & =d\left(\frac{1}{V}\right) \sum_{c=1}^{N} \mathbf{x}^{c} \otimes \mathbf{f}^{c}+\frac{1}{V} \sum_{c=1}^{N} \mathbf{x}^{c} \otimes \dot{\mathbf{f}}^{c}+\frac{1}{V} \sum_{c=1}^{N} \dot{\mathbf{x}}^{c} \otimes \mathbf{f}^{c} \\
& =-(\overline{\boldsymbol{\sigma}} \otimes \mathbf{I}): \dot{\overline{\mathbf{E}}}+\left(\mathbf{D}^{I, \sigma E-1}: \dot{\overline{\mathbf{E}}}+\mathbf{D}^{I, \sigma \mu}: \dot{\overline{\boldsymbol{\mu}}}\right)+\mathbf{D}^{u, \sigma E-2}: \dot{\overline{\mathbf{E}}} \\
& =\mathbf{D}^{I, \sigma E}: \dot{\overline{\mathbf{E}}}+\mathbf{D}^{I, \sigma \mu}: \dot{\overline{\boldsymbol{\mu}}}
\end{aligned}
$$

where the modular tensors are expressed as

$$
\begin{aligned}
& \mathbf{D}^{I, \sigma E}=-(\overline{\boldsymbol{\sigma}} \otimes \mathbf{I})+\mathbf{D}^{I, \sigma E-1}+\mathbf{D}^{u, \sigma E-2} \\
& \mathbf{D}^{I, \sigma E-1}=\frac{1}{V} \sum_{c=1}^{N} \sum_{b=1}^{N} x_{i}^{c} K_{j l}^{I f u(c b)} x_{k}^{b} \mathbf{e}_{i} \otimes \mathbf{e}_{j} \otimes \mathbf{e}_{k} \otimes \mathbf{e}_{l} \\
& \mathbf{D}^{I, \sigma \mu}=\frac{1}{V} \sum_{c=1}^{N} \sum_{b=1}^{N} \Delta S^{b} x_{i}^{c} K_{j l}^{I f m(c b)} n_{k}^{b} \mathbf{e}_{i} \otimes \mathbf{e}_{j} \otimes \mathbf{e}_{k} \otimes \mathbf{e}_{l}
\end{aligned}
$$

As shown in Appendices B.3,B.4,B.5, the three terms at the right hand side of Eq. (70) can be further expressed as

$$
\begin{aligned}
\dot{\overline{\boldsymbol{\kappa}}} & =d\left(\frac{1}{V}\right) \sum_{c=1}^{N} \mathbf{n}^{c} \otimes \boldsymbol{\omega}^{c} \Delta S^{c}+\frac{1}{V} \sum_{c=1}^{N} \mathbf{n}^{c} \otimes \dot{\boldsymbol{\omega}}^{c} \Delta S^{c}+\frac{1}{V} \sum_{c=1}^{N} \dot{\mathbf{n}}^{c} \\
& \otimes \boldsymbol{\omega}^{c} \Delta S^{c} \\
= & -(\overline{\boldsymbol{\kappa}} \otimes \mathbf{I}): \dot{\overline{\mathbf{E}}}+\left(\mathbf{D}^{I, \kappa E-1}: \dot{\overline{\mathbf{E}}}+\mathbf{D}^{I, \kappa \mu-1}: \dot{\overline{\boldsymbol{\mu}}}\right)+\left(\mathbf{D}^{I, \kappa E-2}\right. \\
& \left.: \dot{\overline{\mathbf{E}}}+\mathbf{D}^{I, \kappa \mu-2}: \dot{\overline{\boldsymbol{\mu}}}\right)=\mathbf{D}^{I, \kappa E}: \dot{\overline{\mathbf{E}}}+\mathbf{D}^{I, \kappa \mu}: \dot{\overline{\boldsymbol{\mu}}}
\end{aligned}
$$

where the modular tensors are expressed as

$$
\begin{aligned}
& \mathbf{D}^{I, \kappa E}=-(\overline{\boldsymbol{\kappa}} \otimes \mathbf{I})+\mathbf{D}^{I, \kappa E-1}+\mathbf{D}^{I, \kappa E-2} \\
& \mathbf{D}^{I, \kappa \mu}=\mathbf{D}^{I, \kappa \mu-1}+\mathbf{D}^{I, \kappa \mu-2} \\
& \mathbf{D}^{I, \kappa E-1}=\frac{1}{V} \sum_{c=1}^{N} \sum_{b=1}^{N} \Delta S^{c} n_{i}^{c} K_{j l}^{I \omega u(c b)} x_{k}^{b} \mathbf{e}_{i} \otimes \mathbf{e}_{j} \otimes \mathbf{e}_{k} \otimes \mathbf{e}_{l} \\
& \mathbf{D}^{I, \kappa \mu-1}=\frac{1}{V} \sum_{c=1}^{N} \sum_{b=1}^{N} \Delta S^{c} \Delta S^{b} n_{i}^{c} K_{j l}^{I \omega m(c b)} n_{k}^{b} \mathbf{e}_{i} \otimes \mathbf{e}_{j} \otimes \mathbf{e}_{k} \otimes \mathbf{e}_{l} \\
& \mathbf{D}^{I, \kappa E-2}=\frac{1}{V} \sum_{c=1}^{N} \sum_{b=1}^{N} \Delta S^{c} \omega_{j}^{c} T_{i p}^{n \omega} K_{p l}^{I \omega u(c b)} x_{k}^{b} \mathbf{e}_{i} \otimes \mathbf{e}_{j} \otimes \mathbf{e}_{k} \otimes \mathbf{e}_{l} \\
& \mathbf{D}^{I, \kappa \mu-2}=\frac{1}{V} \sum_{c=1}^{N} \sum_{b=1}^{N} \Delta S^{c} \Delta S^{b} \omega_{j}^{c} T_{i p}^{n \omega} K_{p l}^{I \omega m(c b)} n_{k}^{b} \mathbf{e}_{i} \otimes \mathbf{e}_{j} \otimes \mathbf{e}_{k} \otimes \mathbf{e}_{l}
\end{aligned}
$$

in which $T_{i p}^{n \omega}$ is expressed by Eq. (B.11) in Appendix B.

Eqs. (76) and (80) can be equivalently expressed as

$$
\begin{aligned}
\dot{\overline{\boldsymbol{\sigma}}} & =\mathbf{D}^{I, \sigma E *}: \dot{\overline{\mathbf{E}}}+\mathbf{D}^{I, \sigma \kappa *}: \dot{\overline{\boldsymbol{\kappa}}} \\
\dot{\overline{\boldsymbol{\mu}}} & =\mathbf{D}^{I, \mu E *}: \dot{\overline{\mathbf{E}}}+\mathbf{D}^{I, \mu \kappa *}: \dot{\overline{\boldsymbol{\kappa}}}
\end{aligned}
$$

in which

$$
\begin{aligned}
& \mathbf{D}^{I, \sigma E *}=\mathbf{D}^{I, \sigma E}-\mathbf{D}^{I, \sigma \mu}:\left(\mathbf{D}^{I, \kappa \mu}\right)^{-1}: \mathbf{D}^{I, \kappa E} \\
& \mathbf{D}^{I, \sigma \kappa *}=\mathbf{D}^{I, \sigma \mu}:\left(\mathbf{D}^{I, \kappa \mu}\right)^{-1} \\
& \mathbf{D}^{I, \mu E *}=-\left(\mathbf{D}^{I, \kappa \mu}\right)^{-1}: \mathbf{D}^{I, \kappa E} \\
& \mathbf{D}^{I, \mu \kappa *}=\left(\mathbf{D}^{I, \kappa \mu}\right)^{-1}
\end{aligned}
$$


In summary, Eqs. (87) and (88) are the micromechanically based macroscopic constitutive relations consistent with the mixed boundary conditions I.

\subsection{Constitutive model consistent with the mixed RVE boundary conditions II}

As the mixed boundary conditions II, i.e. $\left.t_{i}\right|_{S}=\left.\left(n_{k} \sigma_{k i}\right)\right|_{S}$ $=n_{k} \bar{\sigma}_{k i},\left.\omega_{i}\right|_{s}=\bar{\omega}_{i}+\bar{\omega}_{i, j} x_{j}$ are imposed on the RVE boundary to ensure the satisfaction of Hill-Mandel condition, the counterpart in the rate form prescribed on the discrete $N$ contacting points of the peripheral particles are written for a typical contacting point $c$ as

$$
\begin{aligned}
& \dot{\mathbf{f}}^{c}=\mathbf{n}^{c} \cdot \dot{\overline{\boldsymbol{\sigma}}} \Delta S^{c} \\
& \dot{\boldsymbol{\omega}}^{c}=\dot{\overline{\boldsymbol{\omega}}}^{+} \mathbf{X}^{c} \cdot \dot{\overline{\boldsymbol{\kappa}}}
\end{aligned}
$$

The macroscopic couple stress rates are expressed by Eq. (45), and we can obtain from Eq. (7)

$\bar{E}_{i j}=\bar{u}_{j, i}=\frac{1}{V} \int_{V} u_{j, i} d V=\frac{1}{V} \int_{S} n_{i} u_{j} d S=\frac{1}{V} \sum_{c=1}^{N} n_{i}^{c} \otimes u_{j}^{c} \Delta S^{c}$

Thus

$$
\begin{aligned}
\dot{\overline{\mathbf{E}}}= & d\left(\frac{1}{V}\right) \sum_{c=1}^{N} \mathbf{n}^{c} \otimes \mathbf{u}^{c} \Delta S^{c}+\frac{1}{V} \sum_{c=1}^{N} \mathbf{n}^{c} \otimes \mathbf{u}^{c} \Delta S^{c}+\frac{1}{V} \sum_{c=1}^{N} \dot{\mathbf{n}}^{c} \\
& \otimes \mathbf{u}^{c} \Delta S^{c}
\end{aligned}
$$

Eqs. (29) and (30) can be equivalently transformed in the following form

$$
\begin{aligned}
& \dot{\mathbf{u}}^{C}=\mathbf{K}^{I I u f} \dot{\mathbf{f}}^{C}+\mathbf{K}^{I l u \omega} \dot{\boldsymbol{\omega}}^{C} \\
& \dot{\mathbf{m}}^{C}=\mathbf{K}^{I I m f} \dot{\mathbf{f}}^{C}+\mathbf{K}^{I I m \omega} \dot{\boldsymbol{\omega}}^{C}
\end{aligned}
$$

in which

$$
\begin{aligned}
& \mathbf{K}^{I l u f}=\left[\mathbf{K}^{f u}\right]^{-1} \\
& \mathbf{K}^{I I u \omega}=-\left[\mathbf{K}^{f u}\right]^{-1} \mathbf{K}^{f \omega} \\
& \mathbf{K}^{\text {IImf }}=\mathbf{K}^{m u} \mathbf{K}^{I I u f} \\
& \mathbf{K}^{I I m \omega}=\mathbf{K}^{m u} \mathbf{K}^{I l u \omega}+\mathbf{K}^{m \omega}
\end{aligned}
$$

Thus we have

$$
\begin{aligned}
& \dot{\mathbf{u}}^{c}=\sum_{b=1}^{N}\left[\mathbf{K}^{I I u f(c b)} \dot{\mathbf{f}}^{b}+\mathbf{K}^{I I u \omega(c b)} \dot{\boldsymbol{\omega}}^{b}\right]
\end{aligned}
$$

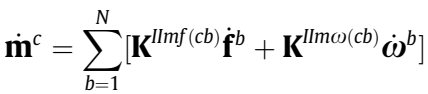

After a series of derivations as shown in Appendices C.1,C.2,C.3, Eq. (92) can be further deduced to result in the following expression

$[\mathbf{I}+(\overline{\mathbf{E}} \otimes \mathbf{I})]: \dot{\overline{\mathbf{E}}}=\mathbf{D}^{I I, E \sigma}: \dot{\overline{\boldsymbol{\sigma}}}+\mathbf{D}^{I I, E \omega} \cdot \dot{\overline{\boldsymbol{\omega}}}+\mathbf{D}^{I I, E \kappa}: \dot{\overline{\boldsymbol{\kappa}}}$

where the modular tensors are expressed as

$$
\begin{aligned}
\mathbf{D}^{I I E \sigma} & =\frac{1}{V} \sum_{c=1}^{N} \sum_{b=1}^{N} \Delta S^{c} \Delta S^{b} n_{i}^{c} K_{j l}^{I I L f(c b)} n_{k}^{b} \mathbf{e}_{i} \otimes \mathbf{e}_{j} \otimes \mathbf{e}_{k} \otimes \mathbf{e}_{l} \\
\mathbf{D}^{I I, E \omega} & =\frac{1}{V} \sum_{c=1}^{N} \sum_{b=1}^{N} \Delta S^{c}\left(n_{i}^{c} K_{j k}^{I I u \omega(c b)}+T_{i l}^{n \omega} u_{j}^{c}\right) \mathbf{e}_{i} \otimes \mathbf{e}_{j} \otimes \mathbf{e}_{k} \\
\mathbf{D}^{I I, E \kappa} & =\frac{1}{V} \sum_{c=1}^{N} \sum_{b=1}^{N} \Delta S^{c}\left(n_{i}^{c} K_{j l}^{I l u \omega(c b)} x_{k}^{b}+T_{i l}^{n \omega} u_{j}^{c} x_{k}^{c}\right) \mathbf{e}_{i} \otimes \mathbf{e}_{j} \otimes \mathbf{e}_{k} \otimes \mathbf{e}_{l}
\end{aligned}
$$

After a series of derivations as shown in Appendices C.4,C.5,C.6, Eq. (45) can be further deduced to result in the following expression
$\dot{\overline{\boldsymbol{\mu}}}=\mathbf{D}^{I I, \mu E}: \dot{\overline{\mathbf{E}}}+\mathbf{D}^{I I, \mu \sigma}: \dot{\overline{\boldsymbol{\sigma}}}+\mathbf{D}^{I I, \mu \omega} \cdot \dot{\overline{\boldsymbol{\omega}}}+\mathbf{D}^{I I, \mu \kappa}: \dot{\overline{\mathcal{\kappa}}}$

where the modular tensors are expressed as

$\mathbf{D}^{I I, \mu E}=-(\overline{\boldsymbol{\mu}} \otimes \mathbf{I}): \dot{\overline{\mathbf{E}}}$

$\mathbf{D}^{I I, \mu \sigma}=\frac{1}{V} \sum_{c=1}^{N} \sum_{b=1}^{N}\left(x_{i}^{c} K_{j l}^{I I m f(c b)}+m_{j}^{c} K_{i l}^{I I L f(c b)}\right) \Delta S^{b} n_{k}^{b} \mathbf{e}_{i} \otimes \mathbf{e}_{j} \otimes \mathbf{e}_{k} \otimes \mathbf{e}_{l}$

$\mathbf{D}^{I I, \mu \omega}=\frac{1}{V} \sum_{c=1}^{N} \sum_{b=1}^{N}\left(x_{i}^{c} K_{j k}^{I I m \omega(c b)}+m_{j}^{c} K_{i k}^{I I L \omega(c b)}\right) \mathbf{e}_{i} \otimes \mathbf{e}_{j} \otimes \mathbf{e}_{k}$

$\mathbf{D}^{I I, \mu \kappa}=\frac{1}{V} \sum_{c=1}^{N} \sum_{b=1}^{N}\left(x_{i}^{c} K_{j l}^{I I m \omega(c b)}+m_{j}^{c} K_{i l}^{I I L \omega(c b)}\right) x_{k}^{b} \mathbf{e}_{i} \otimes \mathbf{e}_{j} \otimes \mathbf{e}_{k} \otimes \mathbf{e}_{l}$

Eqs. (98) and (102) can be further expressed as

$\dot{\overline{\boldsymbol{\sigma}}}=\mathbf{D}^{I I, \sigma E *}: \dot{\overline{\mathbf{E}}}+\mathbf{D}^{I I, \sigma \omega *} \cdot \dot{\overline{\boldsymbol{\omega}}}+\mathbf{D}^{I I, \sigma \kappa *}: \dot{\overline{\boldsymbol{\kappa}}}$

$\dot{\overline{\boldsymbol{\mu}}}=\mathbf{D}^{I I, \mu E *}: \dot{\overline{\mathbf{E}}}+\mathbf{D}^{I I, \mu \omega *} \cdot \dot{\overline{\boldsymbol{\omega}}}+\mathbf{D}^{I I, \mu \kappa *}: \dot{\overline{\boldsymbol{\kappa}}}$

in which

$$
\begin{aligned}
& \mathbf{D}^{I I, \sigma E *}=\left(\mathbf{D}^{I I, E \sigma}\right)^{-1}:[\mathbf{I}+(\overline{\mathbf{E}} \otimes \mathbf{I})] \\
& \mathbf{D}^{I I, \sigma \omega *}=-\left(\mathbf{D}^{I I, E \sigma}\right)^{-1}: \mathbf{D}^{I I, E \omega} \\
& \mathbf{D}^{I I, \sigma \kappa *}=-\left(\mathbf{D}^{I I, E \sigma}\right)^{-1}: \mathbf{D}^{I I, E \kappa} \\
& \mathbf{D}^{I I, \mu E *}=\mathbf{D}^{I I, \mu E}+\mathbf{D}^{I I, \mu \sigma}: \mathbf{D}^{I I, \sigma E *} \\
& \mathbf{D}^{I I, \mu \omega *}=\mathbf{D}^{I I, \mu \omega}+\mathbf{D}^{I I, \mu \sigma}: \mathbf{D}^{I I, \sigma \omega *} \\
& \mathbf{D}^{I I, \mu \kappa *}=\mathbf{D}^{I I, \mu \kappa}+\mathbf{D}^{I I, \mu \sigma}: \mathbf{D}^{I I, \sigma \kappa *}
\end{aligned}
$$

In summary, Eqs. (107) and (108) are the micromechanically based macroscopic constitutive relations consistent with the mixed RVE boundary conditions II.

\subsection{Discussion}

The micromechanically based constitutive models developed in this section are consistent with specific types of RVE boundary conditions which are extracted from Hill's lemma and examined by basic assumptions of the average-field theory [28]. It insures that the developed homogenization scheme satisfies the micromacro energy equivalence condition, i.e. Hill-Mandel condition.

Since the micro and macro models are linked by RVE boundary in the developed homogenization approach, the discrete particle assembly is subjected to RVE boundary displacement and/or traction measures determined from corresponding macroscopic quantities. The mechanical responses of the discrete particle assembly are then analyzed by using discrete element method (DEM) to solve for discrete physical variables, i.e. displacements of each particle and contacting forces between each two particles in contact. Since the equilibrium conditions for each particle are fulfilled in the DEM simulation, the overall equilibrium of the discrete particle assembly RVE is naturally fulfilled, too.

The stiffness matrix $\mathbf{K}_{l}^{n m}$ relating to two typical particles in Eq. (17) can be formulated according to the specific model used on the microscopic level (for example, elastic or elastic-plastic contact models between two particles etc.) to take into account physical evolutions of the microstructures. It is observed from Eqs. (44),(45), (70) and (92) that the evolutions of geometric configuration characterized by the changes of the positions of those particles and the RVE volume are also taken into account in the developed micromechanically based constitutive models.

Three RVE boundary conditions are considered in this paper, as shown in Eqs. (14)-(16). The micromechanically based constitutive Eqs. (67),(68),(87),(88),(107) and (108) can be used to estimate the macroscopic responses under corresponding type of RVE boundary 


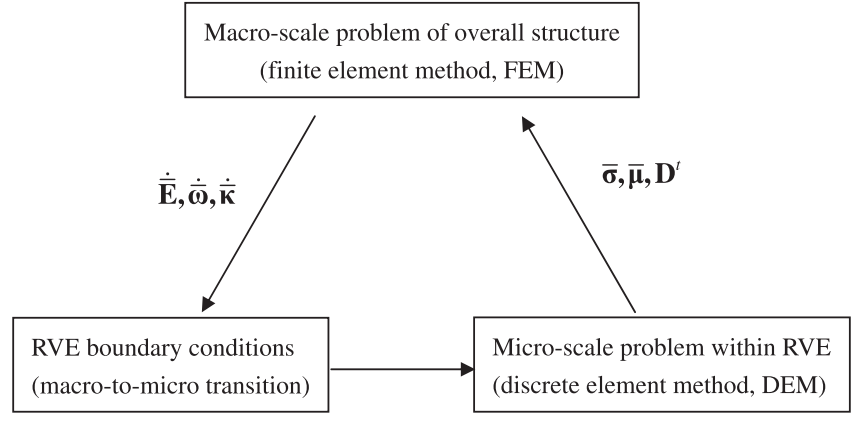

Fig. 5. The computational homogenization scheme.

conditions, respectively. It has been recognized that the homogeneous displacement boundary conditions usually result in a stiff response, while the mixed RVE boundary conditions yield more accurate estimate on the macroscopic responses in the micromacro homogenization method. However, as the computational homogenization procedure is considered, as shown in Fig. 5, the displacement-based finite element method is commonly used on the macroscopic level. The kinematical quantities $\overline{\mathbf{E}}, \overline{\boldsymbol{\omega}}, \overline{\boldsymbol{\kappa}}$ and/or their increments $\overline{\mathbf{E}}, \dot{\overline{\boldsymbol{\omega}}}, \dot{\overline{\boldsymbol{\kappa}}}$ are determined and given at the macroscopic integration points. These kinematical quantities will act as "external loads" to apply to the discrete particle assembly through the RVE boundary. Then, the discrete particles within the RVE will displace and rotate to a new equilibrium status. The stress measures $\overline{\boldsymbol{\sigma}}, \overline{\boldsymbol{\mu}}$ and the tangential modulus matrix $\mathbf{D}^{t}$ at the integration point will be computed and extracted from the discrete particle RVE to be used in the macroscopic finite element equations. This procedure is consistent with the homogeneous displacement RVE boundary conditions Eq. (14) and corresponding micromechanically based constitutive Eqs. (67) and (68).

An alternative way to yield more accurate responses is to construct RVE boundary conditions in the weak form. The periodic boundary conditions on the rectangular-shaped RVE are of this type and have been widely used in the analysis and computation of composites with periodic microstructures [3-7]. Proper periodic RVE boundary conditions of Cosserat continuum have been given in paper [28]. However, granular materials in nature and engineering applications usually do not have periodic microstructure, as shown in Fig. 4. Thus the periodic RVE boundary conditions are not suitable for the physical fact of granular materials. Therefore, the homogeneous displacement RVE boundary conditions are the proper choice for the computational homogenization of granular materials. It can yield stiff, but still reasonable, estimate on the responses of the heterogeneous material.

It should be noted that the stress rate $\dot{\overline{\boldsymbol{\sigma}}}$ and couple stress rate $\dot{\overline{\boldsymbol{\mu}}}$ in the above sections are not objective. As the macroscopic constitutive relations and the associated tangent modular tensors, some objective rates associated with $\dot{\overline{\boldsymbol{\sigma}}}$ and $\dot{\overline{\boldsymbol{\mu}}}$ known as 'co-rotational rates' should be formulated. Alternatively, a specific material reference frame should be specified. We will resort to the co-rotational approach for continua [38-40] in the following work.

\section{Conclusion}

This paper performs further study on the micro-macro homogenization scheme of granular materials [27] based on the advancement of Hill's lemma for Cosserat continuum [28].

Firstly, a new definition of effective couple stress tensor is given for the Cosserat continuum modeling of a discrete particle assembly in light of the average-field theory. Analysis also shows that different definitions of the effective couple stress tensor of granular materials can be used in the homogenization method from the view of the average-field theory, provided that corresponding kinematical quantities are properly chosen to satisfy the micro-macro energy equivalence condition.

Then, rate forms of micromechanically based constitutive models for granular materials are derived according to three types of RVE boundary conditions in the strong form. The micromechanically based constitutive equations can be used to estimate the macroscopic responses under corresponding type of RVE boundary conditions, respectively. Analysis and discussion show that the developed homogenization scheme not only satisfies the micromacro energy equivalence condition and RVE equilibrium condition, but also can take into account the physical and geometrical evolution of the microstructure.

The following work will focus on the application of the presented micro-macro homogenization formulation including the theoretical analysis of effective properties and the numerical simulation of mechanical behavior of granular materials.

\section{Acknowledgement}

The authors are pleased to acknowledge the support of this work by the National Natural Science Foundation of China through the projects with Grant Nos. 11202042 and U1234209.

\section{Appendix A. Derivation of modular tensors under the homogeneous translational and rotational displacement RVE boundary conditions}

\section{A.1. Calculation of $d\left(\frac{1}{V}\right) \sum_{i=1}^{N} \mathbf{x}^{c} \otimes \mathbf{f}^{c}$}

When the rate of change $d\left(\frac{1}{V}\right)$ of the RVE is calculated, the RVE is considered as a continuum and can be expressed as

$d\left(\frac{1}{V}\right)=-\frac{d V}{V^{2}}=-\frac{1}{V^{2}} \operatorname{Vtr}(\dot{\overline{\mathbf{E}}})=-\frac{1}{V} \mathbf{I}: \dot{\mathbf{E}}$

with the macroscopic strain tensor defined as

$\dot{\overline{\mathbf{E}}}=\dot{\bar{E}}_{j i}=\dot{\bar{u}}_{i, j}$

Then we have with the use of Eq. (33)

$d\left(\frac{1}{V}\right) \sum_{c=1}^{N} \mathbf{x}^{c} \otimes \mathbf{f}^{c}=-(\overline{\boldsymbol{\sigma}} \otimes \mathbf{I}): \dot{\overline{\mathbf{E}}}$

\section{A.2. Calculation of $\frac{1}{V} \sum_{c=1}^{N} \mathbf{x}^{c} \otimes \dot{\mathbf{f}}^{c}$}

With Eq. (47) we have

$\bar{V} \sum_{c=1}^{N} \mathbf{x}^{c} \otimes \dot{\mathbf{f}}^{c}=\frac{1}{V} \sum_{c=1}^{N} \mathbf{x}^{c} \otimes \sum_{b=1}^{N}\left[\mathbf{K}^{f u(c b)} \dot{\mathbf{u}}^{b}+\mathbf{K}^{f \omega(c b)} \dot{\boldsymbol{\omega}}^{b}\right]$

Substitution of Eqs. (42) and (43) into Eq. (A.4) leads to

$$
\begin{aligned}
\frac{1}{V} \sum_{c=1}^{N} \mathbf{x}^{c} \otimes \dot{\mathbf{f}}^{c}= & \frac{1}{V} \sum_{c=1}^{N} \mathbf{x}^{c} \otimes \sum_{b=1}^{N}\left[\mathbf{K}^{f u(c b)} \dot{\mathbf{u}}^{b}+\mathbf{K}^{f \omega(c b)} \dot{\boldsymbol{\omega}}^{b}\right] \\
= & \frac{1}{V} \sum_{c=1}^{N} \sum_{b=1}^{N} \mathbf{x}^{c} \otimes\left[\mathbf{K}^{f u(c b)} \cdot\left(\mathbf{x}^{b} \cdot \dot{\overline{\mathbf{E}}}\right)\right]+\frac{1}{V} \sum_{c=1}^{N} \sum_{b=1}^{N} \mathbf{x}^{c} \\
& \otimes\left[\mathbf{K}^{f \omega(c b)} \cdot\left(\dot{\overline{\boldsymbol{\omega}}}+\mathbf{x}^{b} \cdot \dot{\overline{\boldsymbol{\kappa}}}\right)\right] \\
= & \frac{1}{V} \sum_{c=1}^{N} \sum_{b=1}^{N} x_{i}^{c} K_{j l}^{f u(c b)} x_{k}^{b} \dot{\bar{E}}_{k l}+\frac{1}{V} \sum_{c=1}^{N} \sum_{b=1}^{N} x_{i}^{c} K_{j k}^{f \omega(c b)} \dot{\bar{\omega}}_{k} \\
& +\frac{1}{V} \sum_{c=1}^{N} \sum_{b=1}^{N} x_{i}^{c} K_{j l}^{f \omega(c b)} x_{k}^{b} \dot{\bar{\kappa}}_{k l} \\
= & \mathbf{D}^{u, \sigma E-1}: \dot{\overline{\mathbf{E}}}+\mathbf{D}^{u, \sigma \omega} \cdot \dot{\overline{\boldsymbol{\omega}}}+\mathbf{D}^{u, \sigma \kappa}: \dot{\bar{\kappa}}
\end{aligned}
$$


where the modular tensors are expressed as

$\mathbf{D}^{u, \sigma E-1}=\frac{1}{V} \sum_{c=1}^{N} \sum_{b=1}^{N} x_{i}^{c} K_{j l}^{f u(c b)} x_{k}^{b} \mathbf{e}_{i} \otimes \mathbf{e}_{j} \otimes \mathbf{e}_{k} \otimes \mathbf{e}_{l}$

$\mathbf{D}^{u, \sigma \omega}=\frac{1}{V} \sum_{c=1}^{N} \sum_{b=1}^{N} x_{i}^{c} K_{j k}^{f \omega(c b)} \mathbf{e}_{i} \otimes \mathbf{e}_{j} \otimes \mathbf{e}_{k}$

$\mathbf{D}^{u, \sigma \kappa}=\frac{1}{V} \sum_{c=1}^{N} \sum_{b=1}^{N} x_{i}^{c} K_{j l}^{f \omega(c b)} x_{k}^{b} \mathbf{e}_{i} \otimes \mathbf{e}_{j} \otimes \mathbf{e}_{k} \otimes \mathbf{e}_{l}$

\section{A.3. Calculation of $\frac{1}{V} \sum_{c=1}^{N} \dot{\mathbf{x}}^{c} \otimes \mathbf{f}^{c}$}

With the use of boundary Eq. (42), we have

$$
\begin{aligned}
\frac{1}{V} \sum_{c=1}^{N} \dot{\mathbf{x}}^{c} \otimes \mathbf{f}^{c} & =\frac{1}{V} \sum_{c=1}^{N} \dot{\mathbf{u}}^{c} \otimes \mathbf{f}^{c}=\frac{1}{V} \sum_{c=1}^{N}\left(\mathbf{x}^{c} \cdot \dot{\overline{\mathbf{E}}}\right) \otimes \mathbf{f}^{c} \\
& =\frac{1}{V} \sum_{c=1}^{N} \delta_{i l} x_{k}^{c} f_{j}^{c} \dot{\bar{E}}_{k l}=\mathbf{D}^{u, \sigma E-2}: \dot{\overline{\mathbf{E}}}
\end{aligned}
$$

where

$\mathbf{D}^{u, \sigma E-2}=\frac{1}{V} \sum_{c=1}^{N} \delta_{i l} x_{k}^{c} f_{j}^{c} \mathbf{e}_{i} \otimes \mathbf{e}_{j} \otimes \mathbf{e}_{k} \otimes \mathbf{e}_{l}$

\section{A.4. Calculation of $d\left(\frac{1}{V}\right) \sum_{i=1}^{N} \mathbf{x}^{c} \otimes \mathbf{m}^{c}$}

According to Eqs. (A.1),(A.2) and (37), we have

$d\left(\frac{1}{V}\right) \sum_{c=1}^{N} \mathbf{x}^{c} \otimes \mathbf{m}^{c}=-(\overline{\boldsymbol{\mu}} \otimes \mathbf{I}): \dot{\mathbf{E}}$

\section{A.5. Calculation of $\frac{1}{V} \sum_{c=1}^{N} \mathbf{x}^{c} \otimes \dot{\mathbf{m}}^{c}$}

With the use of Eqs. (42),(43) and (48), we have

$$
\begin{aligned}
\frac{1}{V} \sum_{c=1}^{N} \mathbf{x}^{c} \otimes \dot{\mathbf{m}}^{c}= & \frac{1}{V} \sum_{c=1}^{N} \mathbf{x}^{c} \otimes \sum_{b=1}^{N}\left[\mathbf{K}^{m u(c b)} \dot{\mathbf{u}}^{b}+\mathbf{K}^{m \omega(c b)} \dot{\boldsymbol{\omega}}^{b}\right] \\
= & \frac{1}{V} \sum_{c=1}^{N} \sum_{b=1}^{N} \mathbf{x}^{c} \otimes\left[\mathbf{K}^{m u(c b)} \cdot\left(\mathbf{x}^{b} \cdot \dot{\mathbf{E}}\right)\right]+\frac{1}{V} \sum_{c=1}^{N} \sum_{b=1}^{N} \mathbf{x}^{c} \\
& \otimes\left[\mathbf{K}^{m \omega(c b)} \cdot\left(\dot{\overline{\boldsymbol{\omega}}}+\mathbf{x}^{b} \cdot \dot{\overline{\boldsymbol{\kappa}}}\right)\right] \\
= & \frac{1}{V} \sum_{c=1}^{N} \sum_{b=1}^{N} x_{i}^{c} K_{j l}^{m u(c b)} x_{k}^{b} \dot{\bar{E}}_{k l}+\frac{1}{V} \sum_{c=1}^{N} \sum_{b=1}^{N} x_{i}^{c} K_{j k}^{m \omega(c b)} \dot{\bar{\omega}}_{k} \\
& +\frac{1}{V} \sum_{c=1}^{N} \sum_{b=1}^{N} x_{i}^{c} K_{j l}^{m \omega(c b)} x_{k}^{b} \dot{\bar{\kappa}}_{k l} \\
= & \mathbf{D}^{u, \mu E-1}: \dot{\overline{\mathbf{E}}}+\mathbf{D}^{u, \mu \omega} \cdot \dot{\overline{\boldsymbol{\omega}}}+\mathbf{D}^{u, \mu \kappa}: \dot{\bar{\kappa}}
\end{aligned}
$$

where the modular tensors are expressed as

$$
\begin{aligned}
& \mathbf{D}^{u, \mu E-1}=\frac{1}{V} \sum_{c=1}^{N} \sum_{b=1}^{N} x_{i}^{c} K_{j l}^{m u(c b)} x_{k}^{b} \mathbf{e}_{i} \otimes \mathbf{e}_{j} \otimes \mathbf{e}_{k} \otimes \mathbf{e}_{l} \\
& \mathbf{D}^{u, \mu \omega}=\frac{1}{V} \sum_{c=1}^{N} \sum_{b=1}^{N} x_{i}^{c} K_{j k}^{m \omega(c b)} \mathbf{e}_{i} \otimes \mathbf{e}_{j} \otimes \mathbf{e}_{k} \\
& \mathbf{D}^{u, \mu \kappa}=\frac{1}{V} \sum_{c=1}^{N} \sum_{b=1}^{N} x_{i}^{c} K_{j l}^{m \omega(c b)} x_{k}^{b} \mathbf{e}_{i} \otimes \mathbf{e}_{j} \otimes \mathbf{e}_{k} \otimes \mathbf{e}_{l}
\end{aligned}
$$

A.6. Calculation of $\frac{1}{V} \sum_{c=1}^{N} \dot{\mathbf{x}}^{c} \otimes \mathbf{m}^{c}$

With the use of boundary Eq. (42), we have

$$
\begin{aligned}
\frac{1}{V} \sum_{c=1}^{N} \dot{\mathbf{x}}^{c} \otimes \mathbf{m}^{c} & =\frac{1}{V} \sum_{c=1}^{N} \dot{\mathbf{u}}^{c} \otimes \mathbf{m}^{c}=\frac{1}{V} \sum_{c=1}^{N}\left(\mathbf{x}^{c} \cdot \dot{\overline{\mathbf{E}}}\right) \otimes \mathbf{m}^{c} \\
& =\frac{1}{V} \sum_{c=1}^{N} \delta_{i l} x_{k}^{c} m_{j}^{c} \dot{\bar{E}}_{k l}=\mathbf{D}^{u, \mu E-2}: \dot{\overline{\mathbf{E}}}
\end{aligned}
$$

where

$\mathbf{D}^{u, \mu E-2}=\frac{1}{V} \sum_{c=1}^{N} \delta_{i l} x_{k}^{c} m_{j}^{c} \mathbf{e}_{i} \otimes \mathbf{e}_{j} \otimes \mathbf{e}_{k} \otimes \mathbf{e}_{l}$

\section{Appendix B. Derivation of modular tensors under the mixed} RVE boundary conditions I

\section{B.1. Calculation of $d\left(\frac{1}{V}\right) \sum_{i=1}^{N} \mathbf{x}^{c} \otimes \mathbf{f}^{c}$ and $\frac{1}{V} \sum_{c=1}^{N} \dot{\mathbf{x}}^{c} \otimes \mathbf{f}^{c}$}

According to (A.3) and (A.9), we have

$d\left(\frac{1}{V}\right) \sum_{c=1}^{N} \mathbf{x}^{c} \otimes \mathbf{f}^{c}=-(\overline{\boldsymbol{\sigma}} \otimes \mathbf{I}): \dot{\overline{\mathbf{E}}}$

$\frac{1}{V} \sum_{c=1}^{N} \dot{\mathbf{X}}^{c} \otimes \mathbf{f}^{c}=\mathbf{D}^{u, \sigma E-2}: \dot{\overline{\mathbf{E}}}$

\section{B.2. Calculation of $\frac{1}{V} \sum_{c=1}^{N} \mathbf{x}^{c} \otimes \dot{\mathbf{f}}^{c}$}

With the use of Eq. (74), we have

$\frac{1}{V} \sum_{c=1}^{N} \mathbf{x}^{c} \otimes \dot{\mathbf{f}}^{c}=\frac{1}{V} \sum_{c=1}^{N} \mathbf{x}^{c} \otimes \sum_{b=1}^{N}\left[\mathbf{K}^{\mathrm{Ifu}(c b)} \dot{\mathbf{u}}^{b}+\mathbf{K}^{\mathrm{lfm}(c b)} \dot{\mathbf{m}}^{b}\right]$

Substitution of the boundary conditions (69) into Eq. (B.1) gives

$$
\begin{aligned}
\frac{1}{V} \sum_{c=1}^{N} \mathbf{x}^{c} \otimes \dot{\mathbf{f}}^{c} & =\frac{1}{V} \sum_{c=1}^{N} \mathbf{x}^{c} \otimes \sum_{b=1}^{N}\left[\mathbf{K}^{\mathrm{I} f u(c b)} \dot{\mathbf{u}}^{b}+\mathbf{K}^{\mathrm{Ifm}(c b)} \dot{\mathbf{m}}^{b}\right] \\
= & \frac{1}{V} \sum_{c=1}^{N} \sum_{b=1}^{N} \mathbf{x}^{c} \otimes\left[\mathbf{K}^{\mathrm{Ifu}(c b)} \cdot\left(\mathbf{x}^{b} \cdot \dot{\overline{\mathbf{E}}}\right)\right]+\frac{1}{V} \sum_{c=1}^{N} \sum_{b=1}^{N} \mathbf{X}^{c} \\
& \otimes\left[\mathbf{K}^{\mathrm{Ifm}(c b)} \cdot\left(\mathbf{n}^{b} \cdot \dot{\overline{\boldsymbol{\mu}}} \Delta S^{b}\right)\right] \\
= & \frac{1}{V} \sum_{c=1}^{N} \sum_{b=1}^{N} x_{i}^{c} K_{j l}^{\mathrm{Ifu}(c b)} x_{k}^{b} \dot{\bar{E}}_{k l}+\frac{1}{V} \sum_{c=1}^{N} \sum_{b=1}^{N} \Delta S^{b} x_{i}^{c} K_{j l}^{\mathrm{Ifm}(c b)} n_{k}^{b} \dot{\bar{\mu}}_{k l} \\
= & \mathbf{D}^{I, \sigma E-1}: \dot{\overline{\mathbf{E}}}+\mathbf{D}^{I, \sigma \mu}: \dot{\overline{\boldsymbol{\mu}}}
\end{aligned}
$$

where the modular tensors are expressed as

$\mathbf{D}^{I, \sigma E-1}=\frac{1}{V} \sum_{c=1}^{N} \sum_{b=1}^{N} x_{i}^{c} K_{j l}^{I f u(c b)} x_{k}^{b} \mathbf{e}_{i} \otimes \mathbf{e}_{j} \otimes \mathbf{e}_{k} \otimes \mathbf{e}_{l}$

$\mathbf{D}^{I, \sigma \mu}=\frac{1}{V} \sum_{c=1}^{N} \sum_{b=1}^{N} \Delta S^{b} x_{i}^{c} K_{j l}^{I f m(c b)} n_{k}^{b} \mathbf{e}_{i} \otimes \mathbf{e}_{j} \otimes \mathbf{e}_{k} \otimes \mathbf{e}_{l}$

\section{B.3. Calculation of $d\left(\frac{1}{V}\right) \sum_{c=1}^{N} \mathbf{n}^{c} \otimes \omega^{c} \Delta S^{c}$}

According to Eqs. (A.1), (A.2) and (8), we have

$d\left(\frac{1}{V}\right) \sum_{c=1}^{N} \mathbf{n}^{c} \otimes \boldsymbol{\omega}^{c}=-(\overline{\boldsymbol{\kappa}} \otimes \mathbf{I}): \dot{\overline{\mathbf{E}}}$ 
B.4. Calculation of $\frac{1}{V} \sum_{c=1}^{N} \mathbf{n}^{c} \otimes \dot{\omega}^{c} \Delta S^{c}$

With the use of Eqs. (75) and (69), we have

$$
\begin{aligned}
\frac{1}{V} \sum_{c=1}^{N} \mathbf{n}^{c} \otimes \dot{\omega}^{c} \Delta S^{c}= & \frac{1}{V} \sum_{c=1}^{N} \Delta S^{c} \mathbf{n}^{c} \otimes \sum_{b=1}^{N}\left[\mathbf{K}^{I \omega u(c b)} \dot{\mathbf{u}}^{b}+\mathbf{K}^{I \omega m(c b)} \dot{\mathbf{m}}^{b}\right] \\
= & \frac{1}{V} \sum_{c=1}^{N} \sum_{b=1}^{N} \Delta S^{c} \mathbf{n}^{c} \otimes\left[\mathbf{K}^{I \omega u(c b)} \cdot\left(\mathbf{X}^{b} \cdot \dot{\overline{\mathbf{E}}}\right)\right] \\
& +\frac{1}{V} \sum_{c=1}^{N} \sum_{b=1}^{N} \Delta S^{c} \mathbf{n}^{c} \otimes\left[\mathbf{K}^{I \omega m(c b)} \cdot\left(\mathbf{n}^{b} \cdot \dot{\overline{\boldsymbol{\mu}}} \Delta S^{b}\right)\right] \\
= & \frac{1}{V} \sum_{c=1}^{N} \sum_{b=1}^{N} \Delta S^{c} n_{i}^{c} K_{j l}^{I \omega u(c b)} x_{k}^{b} \dot{\bar{E}}_{k l} \\
& +\frac{1}{V} \sum_{c=1}^{N} \sum_{b=1}^{N} \Delta S^{c} \Delta S^{b} n_{i}^{c} K_{j l}^{I \omega m(c b)} n_{k}^{b} \dot{\bar{\mu}}_{k l} \\
= & \mathbf{D}^{I, \kappa E-1}: \dot{\overline{\mathbf{E}}}+\mathbf{D}^{I, \kappa \mu-1}: \dot{\overline{\boldsymbol{\mu}}}
\end{aligned}
$$

where the modular tensors are expressed as

$\mathbf{D}^{I, \kappa E-1}=\frac{1}{V} \sum_{c=1}^{N} \sum_{b=1}^{N} \Delta S^{c} n_{i}^{c} K_{j l}^{I \omega u(c b)} x_{k}^{b} \mathbf{e}_{i} \otimes \mathbf{e}_{j} \otimes \mathbf{e}_{k} \otimes \mathbf{e}_{l}$

$\mathbf{D}^{I, \kappa \mu-1}=\frac{1}{V} \sum_{c=1}^{N} \sum_{b=1}^{N} \Delta S^{c} \Delta S^{b} n_{i}^{c} K_{j l}^{I \omega m(c b)} n_{k}^{b} \mathbf{e}_{i} \otimes \mathbf{e}_{j} \otimes \mathbf{e}_{k} \otimes \mathbf{e}_{l}$

\section{B.5. Calculation of $\frac{1}{V} \sum_{c=1}^{N} \dot{\mathbf{n}}^{c} \otimes \omega^{c} \Delta S^{c}$}

According to Eqs. (105)-(108) in paper [27],

$\dot{\mathbf{n}}^{c}=\left(\mathbf{I}-\mathbf{n}^{c} \otimes \mathbf{n}^{c}\right) \cdot \mathbf{N}^{c} \cdot \dot{\boldsymbol{\omega}}^{c}=\mathbf{T}^{n \omega} \cdot \dot{\boldsymbol{\omega}}^{c}$

where

$\mathbf{N}^{c}=\left[\begin{array}{ccc}0 & n_{3}^{c} & -n_{2}^{c} \\ -n_{3}^{c} & 0 & n_{1}^{c} \\ n_{2}^{c} & -n_{1}^{c} & 0\end{array}\right]$

$\mathbf{T}^{n \omega}=\left(\mathbf{I}-\mathbf{n}^{c} \otimes \mathbf{n}^{c}\right) \cdot \mathbf{N}^{c}$

With the use of Eqs. (75) and (69), we have

$$
\begin{aligned}
\frac{1}{V} \sum_{c=1}^{N} \dot{\mathbf{n}}^{c} \otimes \omega^{c} \Delta S^{c}= & \frac{1}{V} \sum_{c=1}^{N}\left[\mathbf{T}^{n \omega} \cdot \dot{\boldsymbol{\omega}}^{c}\right] \otimes \boldsymbol{\omega}^{c} \Delta S^{c} \\
= & \frac{1}{V} \sum_{c=1}^{N}\left[\mathbf{T}^{n \omega} \cdot \sum_{b=1}^{N}\left(\mathbf{K}^{I \omega u(c b)} \dot{\mathbf{u}}^{b}+\mathbf{K}^{I \omega m(c b)} \dot{\mathbf{m}}^{b}\right)\right] \otimes \boldsymbol{\omega}^{c} \Delta S^{c} \\
= & \frac{1}{V} \sum_{c=1}^{N} \sum_{b=1}^{N}\left[\mathbf{T}^{n \omega} \cdot \mathbf{K}^{I \omega u(c b)} \cdot\left(\mathbf{x}^{b} \cdot \dot{\overline{\mathbf{E}}}\right)\right. \\
& \left.+\mathbf{T}^{n \omega} \cdot \mathbf{K}^{I \omega m(c b)} \cdot\left(\mathbf{n}^{b} \cdot \dot{\overline{\boldsymbol{\mu}}} \Delta S^{b}\right)\right] \otimes \omega^{c} \Delta S^{c} \\
= & \frac{1}{V} \sum_{c=1}^{N} \sum_{b=1}^{N}\left(\Delta S^{c} \omega_{j}^{c} T_{i p}^{n \omega} K_{p l}^{I \omega u(c b)} x_{k}^{b}\right) \dot{\bar{E}}_{k l} \\
& +\frac{1}{V} \sum_{c=1}^{N} \sum_{b=1}^{N}\left(\Delta S^{c} \Delta S^{b} \omega_{j}^{c} T_{i p}^{n \omega} K_{p l}^{I \omega m(c b)} n_{k}^{b}\right) \dot{\bar{\mu}}_{k l} \\
= & \mathbf{D}^{I, \kappa E-2}: \dot{\overline{\mathbf{E}}}+\mathbf{D}^{I, \kappa \mu-2}: \dot{\overline{\boldsymbol{\mu}}}
\end{aligned}
$$

where

$\mathbf{D}^{I, \kappa E-2}=\frac{1}{V} \sum_{c=1}^{N} \sum_{b=1}^{N} \Delta S^{c} \omega_{j}^{c} T_{i p}^{n \omega} K_{p l}^{I \omega u(c b)} x_{k}^{b} \mathbf{e}_{i} \otimes \mathbf{e}_{j} \otimes \mathbf{e}_{k} \otimes \mathbf{e}_{l}$

$\mathbf{D}^{I, \kappa \mu-2}=\frac{1}{V} \sum_{c=1}^{N} \sum_{b=1}^{N} \Delta S^{c} \Delta S^{b} \omega_{j}^{c} T_{i p}^{n \omega} K_{p l}^{I \omega m(c b)} n_{k}^{b} \mathbf{e}_{i} \otimes \mathbf{e}_{j} \otimes \mathbf{e}_{k} \otimes \mathbf{e}_{l}$

\section{Appendix C. Derivation of modular tensors under the mixed RVE boundary conditions II}

C.1. Calculation of $d\left(\frac{1}{V}\right) \sum_{c=1}^{N} \mathbf{n}^{c} \otimes \mathbf{u}^{c} \Delta S^{c}$

According to Eqs. (A.1) and (91), we have

$d\left(\frac{1}{V}\right) \sum_{c=1}^{N} \mathbf{n}^{c} \otimes \mathbf{u}^{c} \Delta S^{c}=-(\overline{\mathbf{E}} \otimes \mathbf{I}): \dot{\overline{\mathbf{E}}}$

\section{C.2. Calculation of $\frac{1}{V} \sum_{c=1}^{N} \mathbf{n}^{c} \otimes \dot{\mathbf{u}}^{c} \Delta S^{c}$}

With the use of Eqs. (90) and (96), we have

$\frac{1}{V} \sum_{c=1}^{N} \mathbf{n}^{c} \otimes \dot{\mathbf{u}}^{c} \Delta S^{c}=\frac{1}{V} \sum_{c=1}^{N} \Delta S^{c} \mathbf{n}^{c} \otimes \sum_{b=1}^{N}\left[\mathbf{K}^{I l u f(c b)} \dot{\mathbf{f}}^{b}+\mathbf{K}^{I l u \omega(c b)} \dot{\boldsymbol{\omega}}^{b}\right]$

$=\frac{1}{V} \sum_{c=1}^{N} \sum_{b=1}^{N} \Delta S^{c} \mathbf{n}^{c} \otimes\left[\mathbf{K}^{I l u f(c b)} \cdot\left(\mathbf{n}^{b} \cdot \dot{\overline{\boldsymbol{\sigma}}} \Delta S^{b}\right)\right]+\frac{1}{V} \sum_{c=1}^{N} \sum_{b=1}^{N} \Delta S^{c} \mathbf{n}^{c} \otimes\left[\mathbf{K}^{I l u \omega(c b)} \cdot\left(\dot{\overline{\boldsymbol{\omega}}}+\mathbf{x}^{b} \cdot \dot{\bar{\kappa}}\right)\right]$

$=\frac{1}{V} \sum_{c=1}^{N} \sum_{b=1}^{N} \Delta S^{c} \Delta S^{b} n_{i}^{c} K_{j l}^{I l u f(c)} n_{k}^{b} \dot{\bar{\sigma}}_{k l}+\frac{1}{V} \sum_{c=1}^{N} \sum_{b=1}^{N} \Delta S^{c} n_{i}^{c} K_{j k}^{I l u \omega(c)} \dot{\bar{\omega}}_{k}+\frac{1}{V} \sum_{c=1}^{N} \sum_{b=1}^{N} \Delta S^{c} n_{i}^{c} K_{j l}^{I l u \omega(c b)} x_{k}^{b} \dot{\bar{\kappa}}_{k l}$

$=\mathbf{D}^{I I, E \sigma}: \dot{\overline{\boldsymbol{\sigma}}}+\mathbf{D}^{I I, E \omega-1} \cdot \dot{\overline{\boldsymbol{\omega}}}+\mathbf{D}^{I I, E \kappa-1}: \dot{\overline{\boldsymbol{\kappa}}}$

where the modular tensors are expressed as

$\mathbf{D}^{I I I E \sigma}=\frac{1}{V} \sum_{c=1}^{N} \sum_{b=1}^{N} \Delta S^{c} \Delta S^{b} n_{i}^{c} K_{j l}^{I I u f(c b)} n_{k}^{b} \mathbf{e}_{i} \otimes \mathbf{e}_{j} \otimes \mathbf{e}_{k} \otimes \mathbf{e}_{l}$

$\mathbf{D}^{I I, E \omega-1}=\frac{1}{V} \sum_{c=1}^{N} \sum_{b=1}^{N} \Delta S^{c} n_{i}^{c} K_{j k}^{I I L \omega(c b)} \mathbf{e}_{i} \otimes \mathbf{e}_{j} \otimes \mathbf{e}_{k}$

$\mathbf{D}^{I I E \kappa-1}=\frac{1}{V} \sum_{c=1}^{N} \sum_{b=1}^{N} \Delta S^{c} n_{i}^{c} K_{j l}^{I I L \omega(c b)} x_{k}^{b} \mathbf{e}_{i} \otimes \mathbf{e}_{j} \otimes \mathbf{e}_{k} \otimes \mathbf{e}_{l}$

\section{C.3. Calculation of $\frac{1}{V} \sum_{c=1}^{N} \dot{\mathbf{n}}^{c} \otimes \mathbf{u}^{c} \Delta S^{c}$}

With Eq. (B.9), we have

$$
\begin{aligned}
\frac{1}{V} \sum_{c=1}^{N} \dot{\mathbf{n}}^{c} \otimes \mathbf{u}^{c} \Delta S^{c} & =\frac{1}{V} \sum_{c=1}^{N} \Delta S^{c}\left[\mathbf{T}^{n \omega} \cdot \dot{\boldsymbol{\omega}}^{c}\right] \otimes \mathbf{u}^{c} \\
& =\frac{1}{V} \sum_{c=1}^{N} \Delta S^{c}\left[\mathbf{T}^{n \omega} \cdot\left(\dot{\overline{\boldsymbol{\omega}}}+\mathbf{x}^{c} \cdot \dot{\bar{\kappa}}\right)\right] \otimes \mathbf{u}^{c} \\
& =\frac{1}{V} \sum_{c=1}^{N}\left(\Delta S^{c} T_{i k}^{n \omega} u_{j}^{c}\right) \dot{\bar{\omega}}_{k}+\frac{1}{V} \sum_{c=1}^{N}\left(\Delta S^{c} T_{i l}^{n \omega} u_{j}^{c} x_{k}^{c}\right) \dot{\bar{\kappa}}_{k l} \\
& =\mathbf{D}^{I I, E \omega-2}: \dot{\overline{\boldsymbol{\omega}}}+\mathbf{D}^{I I, E \kappa-2}: \dot{\bar{\kappa}}
\end{aligned}
$$

where

$$
\begin{aligned}
& \mathbf{D}^{I I, E \omega-2}=\frac{1}{V} \sum_{c=1}^{N} \Delta S^{c} T_{i k}^{n \omega} u_{j}^{c} \mathbf{e}_{i} \otimes \mathbf{e}_{j} \otimes \mathbf{e}_{k} \\
& \mathbf{D}^{I I, E \kappa-2}=\frac{1}{V} \sum_{c=1}^{N} \Delta S^{c} T_{i l}^{n \omega} u_{j}^{c} x_{k}^{c} \mathbf{e}_{i} \otimes \mathbf{e}_{j} \otimes \mathbf{e}_{k} \otimes \mathbf{e}_{l}
\end{aligned}
$$

Thus, we have

$$
\begin{aligned}
\dot{\overline{\mathbf{E}}} & =d\left(\frac{1}{V}\right) \sum_{c=1}^{N} \mathbf{n}^{c} \otimes \mathbf{u}^{c} \Delta S^{c}+\frac{1}{V} \sum_{c=1}^{N} \mathbf{n}^{c} \otimes \dot{\mathbf{u}}^{c} \Delta S^{c}+\frac{1}{V} \sum_{c=1}^{N} \dot{\mathbf{n}}^{c} \\
& \otimes \mathbf{u}^{c} \Delta S^{c} \\
& =-(\overline{\mathbf{E}} \otimes \mathbf{I}): \dot{\overline{\mathbf{E}}}+\mathbf{D}^{I I, E \sigma}: \dot{\overline{\boldsymbol{\sigma}}}+\mathbf{D}^{I I E \omega \omega-1} \cdot \dot{\overline{\boldsymbol{\omega}}}+\mathbf{D}^{I I E \kappa-1} \\
& : \dot{\overline{\boldsymbol{\kappa}}}+\mathbf{D}^{I I I E \omega-2}: \dot{\overline{\boldsymbol{\omega}}}+\mathbf{D}^{I I, E \kappa-2}: \dot{\overline{\boldsymbol{\kappa}}}
\end{aligned}
$$

i.e.

$[\mathbf{I}+(\overline{\mathbf{E}} \otimes \mathbf{I})]: \dot{\overline{\mathbf{E}}}=\mathbf{D}^{I I, E \sigma}: \dot{\overline{\boldsymbol{\sigma}}}+\mathbf{D}^{I I, E \omega} \cdot \dot{\overline{\boldsymbol{\omega}}}+\mathbf{D}^{I I, E \kappa}: \dot{\overline{\boldsymbol{\kappa}}}$ 
$\mathbf{D}^{I I, E \omega}=\mathbf{D}^{I I I E \omega-1}+\mathbf{D}^{I I, E \omega-2}$

$\mathbf{D}^{I I, E \kappa}=\mathbf{D}^{I I, E \kappa-1}+\mathbf{D}^{I I, E \kappa-2}$

\section{C.4. Calculation of $d\left(\frac{1}{V}\right) \sum_{i=1}^{N} \mathbf{x}^{c} \otimes \mathbf{m}^{c}$}

According to Eqs. (A.1) and (37), we have

$d\left(\frac{1}{V}\right) \sum_{c=1}^{N} \mathbf{x}^{c} \otimes \mathbf{m}^{c}=-(\overline{\boldsymbol{\mu}} \otimes \mathbf{I}): \dot{\overline{\mathbf{E}}}$

\section{C.5. Calculation of $\frac{1}{V} \sum_{c=1}^{N} \mathbf{x}^{c} \otimes \dot{\mathbf{m}}^{c}$}

With the use of Eqs. (90) and (97), we have

$$
\begin{aligned}
\frac{1}{V} \sum_{c=1}^{N} \mathbf{X}^{c} \otimes \dot{\mathbf{m}}^{c}= & \frac{1}{V} \sum_{c=1}^{N} \mathbf{X}^{c} \otimes \sum_{b=1}^{N}\left[\mathbf{K}^{\operatorname{IImf}(c b)} \dot{\mathbf{f}}^{b}+\mathbf{K}^{\operatorname{IIm} \omega(c b)} \dot{\boldsymbol{\omega}}^{b}\right] \\
= & \frac{1}{V} \sum_{c=1}^{N} \sum_{b=1}^{N} \mathbf{x}^{c} \otimes\left[\mathbf{K}^{\operatorname{IImf}(c b)} \cdot\left(\mathbf{n}^{b} \cdot \dot{\overline{\boldsymbol{\sigma}}} \Delta S^{b}\right)\right] \\
& +\frac{1}{V} \sum_{c=1}^{N} \sum_{b=1}^{N} \mathbf{x}^{c} \otimes\left[\mathbf{K}^{\operatorname{IIm} \omega(c b)} \cdot\left(\dot{\overline{\boldsymbol{\omega}}}+\mathbf{x}^{b} \cdot \dot{\overline{\boldsymbol{\kappa}}}\right)\right] \\
= & \frac{1}{V} \sum_{c=1}^{N} \sum_{b=1}^{N} \Delta S^{b} x_{i}^{c} K_{j l}^{\operatorname{IIm}(c b)} n_{k}^{b} \dot{\bar{\sigma}}_{k l}+\frac{1}{V} \sum_{c=1}^{N} \sum_{b=1}^{N} x_{i}^{c} K_{j k}^{I I m \omega(c b)} \dot{\bar{\omega}}_{k} \\
& +\frac{1}{V} \sum_{c=1}^{N} \sum_{b=1}^{N} x_{i}^{c} K_{j l}^{\operatorname{IIm} \omega(c b)} x_{k}^{b} \dot{\bar{\kappa}}_{k l} \\
= & \mathbf{D}^{I I, \mu \sigma-1}: \dot{\overline{\boldsymbol{\sigma}}}+\mathbf{D}^{I I, \mu \omega-1} \cdot \dot{\overline{\boldsymbol{\omega}}}+\mathbf{D}^{I I, \mu \kappa-1}: \dot{\overline{\boldsymbol{\kappa}}}
\end{aligned}
$$

where the modular tensors are expressed as

$$
\begin{aligned}
& \mathbf{D}^{I I, \mu \sigma-1}=\frac{1}{V} \sum_{c=1}^{N} \sum_{b=1}^{N} \Delta S^{b} x_{i}^{c} K_{j l}^{I I m f(c b)} n_{k}^{b} \mathbf{e}_{i} \otimes \mathbf{e}_{j} \otimes \mathbf{e}_{k} \otimes \mathbf{e}_{l} \\
& \mathbf{D}^{I I, \mu \omega-1}=\frac{1}{V} \sum_{c=1}^{N} \sum_{b=1}^{N} x_{i}^{c} K_{j k}^{I I m \omega(c b)} \mathbf{e}_{i} \otimes \mathbf{e}_{j} \otimes \mathbf{e}_{k} \\
& \mathbf{D}^{I I, \mu \kappa-1}=\frac{1}{V} \sum_{c=1}^{N} \sum_{b=1}^{N} x_{i}^{c} K_{j l}^{I I m \omega(c b)} x_{k}^{b} \mathbf{e}_{i} \otimes \mathbf{e}_{j} \otimes \mathbf{e}_{k} \otimes \mathbf{e}_{l}
\end{aligned}
$$

\section{C.6. Calculation of $\frac{1}{V} \sum_{c=1}^{N} \dot{\mathbf{x}}^{c} \otimes \mathbf{m}^{c}$}

With the use of Eqs. (90) and (96), we have

$$
\begin{aligned}
& \frac{1}{V} \sum_{c=1}^{N} \dot{\mathbf{x}}^{c} \otimes \mathbf{m}^{c}=\frac{1}{V} \sum_{c=1}^{N} \dot{\mathbf{u}}^{c} \otimes \mathbf{m}^{c} \\
& =\frac{1}{V} \sum_{c=1}^{N} \sum_{b=1}^{N}\left[\mathbf{K}^{I I u f(c b)} \dot{\mathbf{f}}^{b}+\mathbf{K}^{I I u \omega(c b)} \dot{\boldsymbol{\omega}}^{b}\right] \otimes \mathbf{m}^{c} \\
& =\frac{1}{V} \sum_{c=1}^{N} \sum_{b=1}^{N}\left[\mathbf{K}^{I I u f(c b)} \cdot\left(\mathbf{n}^{b} \cdot \dot{\overline{\boldsymbol{\sigma}}} \Delta S^{b}\right)\right. \\
& \left.+\mathbf{K}^{I I u \omega(c b)} \cdot\left(\dot{\overline{\boldsymbol{\omega}}}+\mathbf{X}^{b} \cdot \dot{\overline{\boldsymbol{\kappa}}}\right)\right] \otimes \mathbf{m}^{c} \\
& =\frac{1}{V} \sum_{c=1}^{N} \sum_{b=1}^{N} \Delta S^{b} m_{j}^{c} K_{i l}^{I I u f(c b)} n_{k}^{b} \dot{\bar{\sigma}}_{k l} \\
& +\frac{1}{V} \sum_{c=1}^{N} \sum_{b=1}^{N} m_{j}^{c} K_{i k}^{I l u \omega(c b)} \dot{\bar{\omega}}_{k}+\frac{1}{V} \sum_{c=1}^{N} \sum_{b=1}^{N} m_{j}^{c} K_{i l}^{I l u \omega(c b)} x_{k}^{b} \dot{\overline{\boldsymbol{\kappa}}}_{k l} \\
& =\mathbf{D}^{I I, \mu \sigma-2}: \dot{\overline{\boldsymbol{\sigma}}}+\mathbf{D}^{I I, \mu \omega-2} \cdot \dot{\overline{\boldsymbol{\omega}}}+\mathbf{D}^{I I, \mu \kappa-2}: \dot{\overline{\boldsymbol{\kappa}}}
\end{aligned}
$$

where

$$
\begin{aligned}
& \mathbf{D}^{I I, \mu \sigma-2}=\frac{1}{V} \sum_{c=1}^{N} \sum_{b=1}^{N} \Delta S^{b} m_{j}^{c} K_{i l}^{I I L f(c b)} n_{k}^{b} \mathbf{e}_{i} \otimes \mathbf{e}_{j} \otimes \mathbf{e}_{k} \otimes \mathbf{e}_{l} \\
& \mathbf{D}^{I I, \mu \omega-2}=\frac{1}{V} \sum_{c=1}^{N} \sum_{b=1}^{N} m_{j}^{c} K_{i k}^{I I L \omega(c b)} \mathbf{e}_{i} \otimes \mathbf{e}_{j} \otimes \mathbf{e}_{k} \\
& \mathbf{D}^{I I, \mu \kappa-2}=\frac{1}{V} \sum_{c=1}^{N} \sum_{b=1}^{N} m_{j}^{c} K_{i l}^{I I L \omega(c b)} x_{k}^{b} \mathbf{e}_{i} \otimes \mathbf{e}_{j} \otimes \mathbf{e}_{k} \otimes \mathbf{e}_{l}
\end{aligned}
$$

So, we have

$$
\begin{aligned}
\dot{\overline{\boldsymbol{\mu}}} & =d\left(\frac{1}{V}\right) \sum_{c=1}^{N} \mathbf{x}^{c} \otimes \mathbf{m}^{c}+\frac{1}{V} \sum_{c=1}^{N} \mathbf{x}^{c} \otimes \dot{\mathbf{m}}^{c}+\frac{1}{V} \sum_{c=1}^{N} \dot{\mathbf{x}}^{c} \otimes \mathbf{m}^{c} \\
& =-(\overline{\boldsymbol{\mu}} \otimes \mathbf{I}): \dot{\overline{\mathbf{E}}}+\mathbf{D}^{I I, \mu \sigma-1}: \dot{\overline{\boldsymbol{\sigma}}}+\mathbf{D}^{I I, \mu \omega-1} \cdot \dot{\overline{\boldsymbol{\omega}}}+\mathbf{D}^{I I, \mu \kappa-1} \\
& : \dot{\overline{\boldsymbol{\kappa}}}+\mathbf{D}^{I I, \mu \sigma-2}: \dot{\overline{\boldsymbol{\sigma}}}+\mathbf{D}^{I I, \mu \omega-2} \cdot \dot{\overline{\boldsymbol{\omega}}}+\mathbf{D}^{I I, \mu \kappa-2}: \dot{\overline{\boldsymbol{\kappa}}}=\mathbf{D}^{I I, \mu E} \\
& : \dot{\overline{\mathbf{E}}}+\mathbf{D}^{I I, \mu \sigma}: \dot{\overline{\boldsymbol{\sigma}}}+\mathbf{D}^{I I, \mu \omega} \cdot \dot{\overline{\boldsymbol{\omega}}}+\mathbf{D}^{I I, \mu \kappa}: \dot{\overline{\boldsymbol{\kappa}}}
\end{aligned}
$$

in which

$$
\begin{aligned}
& \mathbf{D}^{I I, \mu E}=-(\overline{\boldsymbol{\mu}} \otimes \mathbf{I}) \\
& \mathbf{D}^{I I, \mu \sigma}=\mathbf{D}^{I I, \mu \sigma-1}+\mathbf{D}^{I I, \mu \sigma-2} \\
& \mathbf{D}^{I I, \mu \omega}=\mathbf{D}^{I I, \mu \omega-1}+\mathbf{D}^{I I, \mu \omega-2} \\
& \mathbf{D}^{I I, \mu \kappa}=\mathbf{D}^{I I, \mu \kappa-1}+\mathbf{D}^{I I, \mu \kappa-2}
\end{aligned}
$$

\section{References}

[1] R. Hill, Elastic properties of reinforced solids: some theoretical principles, Journal of the Mechanics and Physics in Solids 11 (1963) 357-372.

[2] Z. Hashin, Analysis of composite materials - a survey, Journal of Applied Mechanics 50 (1983) 481-505.

[3] P.M. Suquet, Local and global aspects in the mathematical theory of plasticity, in: A. Sawczuk, G. Bianchi (Eds.), Plasticity Today: Modelling, Methods and Applications, Elsevier Applied Science Publishers, London, 1985, pp. 279-310.

[4] S. Nemat-Nasser, M. Hori, Micromechanics: Overall Properties of Heterogeneous Materials, Elsevier, Amsterdam, 1999.

[5] J.C. Michel, C. Moulinec, P.M. Suquet, Effective properties of composite materials with periodic macrostructure: a computational approach, Computer Methods in Applied Mechanics and Engineering 172 (1999) 109143.

[6] C. Miehe, A. Koch, Computational micro-to-macro transitions of discretized microstructures undergoing small strains, Archive of Applied Mechanics 72 (2002) 300-317.

[7] J. Qu, M. Cherkaoui, Fundamentals of Micromechanics of Solids, John Wiley \& Sons, Hoboken New Jersey, 2006.

[8] S. Yuu, T. Umekage, Constitutive relations and computer simulation of granular material, Advanced Powder Technology 19 (2008) 203-230.

[9] Q. Zhou, H.W. Zhang, Y.G. Zheng, A homogenization technique for heat transfer in periodic granular materials, Advanced Powder Technology 23 (2012) 104114.

[10] M. Oda, K. Iwashita, Mechanics of Granular Materials, A.A. Ballkema, Rotterdam, 1999.

[11] M.J. Jiang, H.S. Yu, D. Harris, A novel discrete model for granular material incorporating rolling resistance, Computers and Geotechnics 32 (2005) 340 357.

[12] H.B. Muhlhaus, I. Vardoulakis, The thickness of shear bands in granular materials, Geotechnique 37 (1987) 271-283.

[13] A. Tordesillas, S.D.C. Walsh, Incorporating rolling resistance and contact anisotropy in micromechanical models of granular media, Powder Technology 124 (2002) 106-111.

[14] C.S. Chang, M.R. Kuhn, On virtual work and stress in granular media International Journal of Solids and Structures 42 (2005) 3773-3793.

[15] J.D. Goddard, From granular matter to generalized continuum, in: G. Capriz, P. Giovine, P.M. Mariano (Eds.), Mathematical Models of Granular Matter, Lecture Notes in Applied Mathematics, Springer-Verlag, Berlin, 2008, pp. 1-20.

[16] R. de Borst, Simulation of strain localization: a reappraisal of the Cosserat continuum, Engineering Computations 8 (1991) 317-332.

[17] T. Ebinger, H. Steeb, S. Diebels, Modeling macroscopic extended continua with the aid of numerical homogenization schemes, Computational Material Science 32 (2005) 337-347.

[18] X.K. Li, H.X. Tang, A consistent return mapping algorithm for pressuredependent elastoplastic Cosserat continua and modeling of strain localization, Computers and Structures 83 (2005) 1-10.

[19] M.I. Alsaleh, G.Z. Voyiadjis, K.A. Alshibli, Modeling strain localization in granular materials using micropolar theory: mathematical formulations, 
International Journal for Numerical and Analytical Methods in Geomechanics 30 (2006) 1501-1524.

[20] H. Arslan, S. Sture, Evaluation of a physical length scale for granular materials, Computational Material Science 42 (2008) 525-530.

[21] S. Forest, R. Dendievel, G.R. Canova, Estimating the overall properties of heterogeneous Cosserat materials, Modelling and Simulation in Materia Science and Engineering 7 (1999) 829-840.

[22] X. Yuan, Y. Tomita, Effective properties of Cosserat composites with periodic microstructure, Mechanics Research Communication 28 (2001) 265-270.

[23] G.K. Hu, X.N. Liu, T.J. Lu, A variational method for non-linear micropolar composites, Mechanics of Materials 37 (2005) 407-425

[24] J. Sulem, H.B. Mühlhaus, A continuum model for periodic two-dimensional block structures, Mechanics of Cohesive-Frictional Materials 2 (1997) 31-46.

[25] P. Trovalusci, R. Masiani, Non-linear micropolar and classical continua for anisotropic discontinuous materials, International Journal of Solids and Structures 40 (2003) 1281-1297.

[26] D. Besdo, Towards a Cosserat-theory describing motion of an originally rectangular structure of blocks, Archive of Applied Mechanics 80 (2010) 2545.

[27] X.K. Li, Q.P. Liu, J. Zhang, A micro-macro homogenization approach of discrete particle assembly - Cosserat continuum modeling of granular materials, International Journal of Solids and Structures 47 (2010) 291-303.

[28] Q. P Liu, Hill's lemma for the average-field theory of Cosserat continuum, Acta Mechanica 224 (2013) 851-866.

[29] R.D. Mindlin, Micro-structure in linear elasticity, Archive for Rational Mechanics and Analysis 16 (1964) 51-78.

[30] N.A. Fleck, J.W. Hutchinson, Strain gradient plasticity, Advances in Applied Mechanics 33 (1997) 295-361.
[31] P.A. Cundall, O.D.L. Strack, A discrete numerical model for granular assemblies, Geotechnique 29 (1979) 47-65.

[32] X.K. Li, X.H. Chu, Y.T. Feng, A discrete particle model and numerical modeling of the failure modes of granular materials, Engineering Computations 22 (2005) 894-920.

[33] M.R. Kuhn, C.S. Chang, Stability, bifurcation, and softening in discrete systems: a conceptual approach for granular materials, International Journal of Solids and Structures 43 (2006) 6026-6051.

[34] K. Bagi, On the concept of jammed configurations from a structural mechanics perspective, Granular Matter 9 (2007) 109-134.

[35] A.S.J. Suiker, R. de Borst, C.S. Chang, Micro-mechanical modelling of granular material, Part I: Derivation of a second-gradient micro-polar constitutive theory, Acta Mechanica 149 (2001) 161-181.

[36] J.P. Bardet, I. Vardoulakis, The asymmetry of stress in granular media, International Journal of Solids and Structures 38 (2001) 353-367.

[37] N.P. Kruyt, Statics and kinematics of discrete Cosserat-type granular materials, International Journal of Solids and Structures 40 (2003) 511-534.

[38] M.A. Crisfield, G.F. Moita, A co-rotational formulation for 2-D continua including incompatible modes, International Journal for Numerical Methods in Engineering 39 (1996) 2619-2633.

[39] P.H. Jetteur, S. Cescotto, Mixed finite element for the analysis of large inelastic strains, International Journal for Numerical Methods in Engineering 31 (1991) 229-239.

[40] X.K. Li, S. Cescotto, Finite element method for gradient plasticity at large strains, International Journal for Numerical Methods in Engineering 39 (1996) 619-633. 